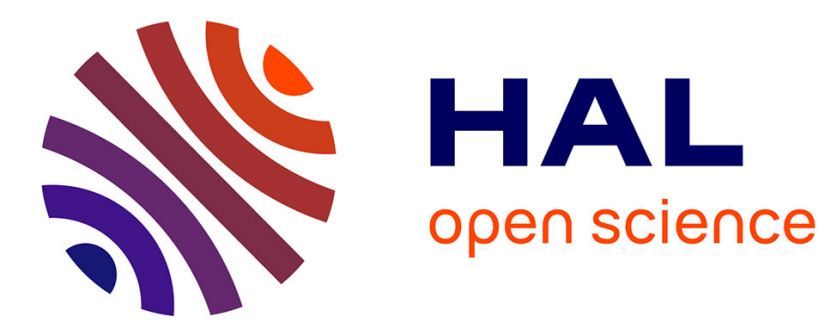

\title{
Phonon effects on X-ray absorption and X-ray photoemission spectra
}

Takashi Fujikawa, Hiroto Sakuma, Kaori Niki, Didier Sébilleau

\section{To cite this version:}

Takashi Fujikawa, Hiroto Sakuma, Kaori Niki, Didier Sébilleau. Phonon effects on X-ray absorption and X-ray photoemission spectra. Journal of Electron Spectroscopy and Related Phenomena, 2015, 198, pp.57-67. 10.1016/j.elspec.2014.12.002 . hal-01109966

\section{HAL Id: hal-01109966 https://hal-univ-rennes1.archives-ouvertes.fr/hal-01109966}

Submitted on 27 Jan 2015

HAL is a multi-disciplinary open access archive for the deposit and dissemination of scientific research documents, whether they are published or not. The documents may come from teaching and research institutions in France or abroad, or from public or private research centers.
L'archive ouverte pluridisciplinaire HAL, est destinée au dépôt et à la diffusion de documents scientifiques de niveau recherche, publiés ou non, émanant des établissements d'enseignement et de recherche français ou étrangers, des laboratoires publics ou privés. 
Research Highlight

Some important phonon effects observed in X-ray absorption and $\mathrm{X}$-ray photoemis- sion spectra are discussed on the basis of nonequilibrium Green's function theory. For the pre-edge structures, the intensity associated with forbidden electric dipole transition is sensitive to temperature compared with allowed electric quadrupole transition. We also discuss the FC and their interference, which have negligible contribution to pre- edge intensity and energy shift. The quasi-particle energy is also influenced by the core displacement which can be responsible for the peak shift of the pre-edges. We also discuss the photoelectron angular distribution caused by the thermal atomic vibration. 


\title{
Phonon Effects on X-ray Absorption and X-ray Photoemission Spectra
}

\author{
Takashi Fujikawa ${ }^{a}$,Hiroto Sakuma ${ }^{a}$,Kaori Niki ${ }^{a}$ \\ Didier Sébilleau ${ }^{\mathrm{b}}$ \\ ${ }^{a}$ Graduate School of Advanced Integration Science, Chiba University, 1-33 \\ Yayoi-cho, Inage-ku, Chiba 263-8522, Japan \\ ${ }^{\mathrm{b}}$ Département Matériaux-Nanosciences, Institut de Physique de Rennes, UMR \\ UR1-CNRS 6251, Campus de Beaulieu, Universite de Rennes1, 35042 \\ Rennes-cedex, France
}

\begin{abstract}
Some important phonon effects observed in X-ray absorption and X-ray photoemission spectra are discussed on the basis of nonequilibrium Green's function theory. This theoretical framework allows us to incorporate phonon effects, such as DebyeWaller (DW) factors, Franck-Condon (FC) factors and electron-phonon interactions in a natural way. In the case of core level excitations, we can take into account the core-hole effects in lesser Green's function $g^{<}$and photoelectron propagation in greater Green's function $g^{>}$. For the core-hole propagation we derive some formulas to describe the thermally displaced core functions: we have $p$ components even for deep core $s$ orbital due to the thermal motion. We should notice that the thermal fluctuation is quite small but it is already in the order of the spread of the core functions. Applying Mermin's theorem, we can calculate the thermal average of the hole propagator $g^{<}$: Here an important ingredient is the Debye-Waller factor used in $\mathrm{X}$-ray and neutron diffraction. For the pre-edge structures, the intensity associated with forbidden electric dipole transition is sensitive to the temperature compared with allowed electric quadrupole transition. We also discuss the FC and their interference, which have negligible contribution to pre-edge intensity and energy shift. The quasi-particle energy is also influenced by the core displacement which can be responsible for the peak shift of the pre-edges. We also discuss the changes of the photoelectron angular distributions caused by the thermal atomic vibration.
\end{abstract}

Key words: XPS, XAFS, electron-phonon interaction, Debye-Waller factors, Franck-Condon factors, nonequilibrium Green's function, pre-edge structures, core displacement

Email address: tfujikawa@faculty.chiba-u.jp (Takashi Fujikawa). 


\section{Introduction}

Among phonon effects on X-ray absorption spectra, the EXAFS Debye-Waller (DW) factors have extensively been studied, whereas other factors like FranckCondon (FC) factors and electron-phonon interactions are rarely discussed. Within the single-scattering EXAFS theory, both the DW and FC and their interference terms are explicitly taken into account [1,2]. A more sophisticated approach including electron-phonon interaction has been developed on the basis of Keldysh Green's function formalism [3]. • • Ankudinov and Rehr have shown that local atomic displacements are responsible for additional XANES peaks [4]. They used a simple formula for the X-ray absorption intensity $I(\omega, Q)$ considering the thermal average shown by $\langle\ldots\rangle$

$$
\begin{aligned}
& <I(\omega, Q)>=I\left(\omega, Q^{0}\right)+\frac{1}{2} \sum_{\alpha i} \sum_{\beta j}<u_{\alpha i} u_{\beta j}>\left.\frac{\partial^{2} I(\omega, Q)}{\partial u_{\alpha i} \partial u_{\beta j}}\right|_{0} \\
& (i, j=x, y, z)
\end{aligned}
$$

where $Q$ designates the assembly of nuclei $Q=\left(\mathbf{R}_{\alpha}, \mathbf{R}_{\beta}, \ldots\right)$. The above equation takes small deviation from equilibrium atomic configuration $Q^{0}(u=$ $\left.Q-Q^{0}\right)$. The first order terms cancel since $\left\langle u_{\alpha i}\right\rangle=0$. For solids the summation over $\alpha$ and $\beta$ runs over all composite atoms. Prominent temperature dependence of the pre-edge structures are observed in Ti K-edge XANES in $\mathrm{SrTiO}_{3}$ from 15 to 300K: One of the pre-edge peaks shows an increase of the intensity with temperature as observed by Nozawa et al. [5]. More recently Manuel et al. present the Al K-edge XANES spectra of corundum and beryl for temperature range from 300 to $930 \mathrm{~K}$ [6]. These experimental results provide an evidence of the role of thermal fluctuation in XANES at the Al K edge: The pre-edge grows and shifts to lower energy with temperature. They use first principle density functional theory (DFT) calculations for both compounds. They demonstrate that the pre-edge peak originates from forbidden $1 s \rightarrow 3 s$ transition. The theoretical analyses used there are based on their previous papers $[7,8]$. The electron-phonon interaction in XAFS spectra has been studied on the basis of nonrelativistic Keldysh Green's function theory by Arai et al. [3], there they discuss the inelastic mean free path of electrons due to the electron-phonon interaction in XANES spectra.

There is also a growing interest in phonon effects on photoemission spectra. Plucinski et al. study the temperature dependence of W(110) soft X-ray angle resolved photoemission spectra excited at $\omega=260-870 \mathrm{eV}$ and $T=300-780 \mathrm{~K}$ [9]. They have shown that meaningful band mapping can be done for the test case of $\mathrm{W}$ at room temperature with photon energies up to $870 \mathrm{eV}$. Highenergy XPS spectra probe more accurately bulk rather than surface electronic structures, but some questions are raised in regard to the possibility of band 
mapping. Osterwalder et al. report the photoelectron angular distribution from $\mathrm{A} l$ (001) valence band excited at $\omega=1254 \mathrm{eV}$ which presents strong maxima of intensity at main crystallographic directions resembling very closely to the angular distribution of photoelectrons from the $2 s$ core level [10]. The occurrence of maxima of intensity at main interatomic directions is a well-known phenomenon in core-level XPS because of forward focusing [11]. Vicent Alvarez et al. proposed a model to analyze these phenomena based on oneelectron theory [12]. They successfully explain the interesting behavior of the angular distributions of photoelectrons, however, it is hard to estimate the relative importance of Debye-Waller (DW), Franck-Condon (FC) factors, and electron-phonon interaction in that one-electron theory.

Recent experimental work by Takata et al. clearly shows that $\mathrm{C} 1 \mathrm{~s}$ peak shift to high binding energy side with increase of photon energy [13]. The energy shift is well explained by

$$
\Delta \varepsilon_{p}=-p^{2} /\left(2 M_{A}\right)
$$

where $M_{A}$ is the mass of an X-ray absorbing atom $A$. This is just classical free atom recoil energy shift. From physical point of view, this result looks so funny because the X-ray absorbing atom is tightly bound in solids. The above recoil energy shift and peak broadening are successfully explained by considering phonon excitation [14-16]. Recoil effects in valence band photoemission of organic solids have been studied by Shang et al. [17,18]: Specific features sensitive to photon energy are well explained by use of Gelius formula [19] and harmonic approximation for the atomic vibration.

The FC factors have also been discussed for XPS [20] and EXAFS analyses $[1,2]$. In EXAFS the FC factors play a minor role, whereas in XPS they contribute to the temperature dependent peak broadenings. In contrast the influence of electron-phonon interactions on the photoemission spectra are discussed only within the sudden approximation in the UPS region [21,22]. A first principle theory is recently proposed [23] to handle the phonon effects such as DW, FC factors and electron-phonon interactions on photoemission spectra from deep cores, and also extended shallow valence levels based on Keldysh Green's function theory [24] and the Baym-Hedin approach [25,26]. Quite recently Braun et al. present a one-step relativistic theory of temperature dependent photoemission, where phonon effects are explicitly taken into account [27]. These first principle theories, however, have neglected core displacement effects on core excitation. Here we give an approach to incorporate those effects in the Keldysh Green's function theory to discuss the phonon effects on XAFS and XPS spectra, since we can include those temperature effects in a natural way. 


\section{Phonon Effects on XAFS}

In this section we discuss phonon effects on XAFS spectra based on Keldysh Green's function formalism taking atomic thermal displacements.

\subsection{General Consideration}

In the Keldysh Green's function approach the X-ray absorption intensity is described by use of the reducible polarization propagator $\pi^{>}(1,2)[28,29]$. The absorption intensity for X-ray photon with energy $\omega$ is then given by

$$
I(\omega)=-\operatorname{Im}\left[\int d x d x^{\prime} \Delta^{*}(x) \Delta\left(x^{\prime}\right) \int_{-\infty}^{\infty} \pi^{>}\left(x t, x^{\prime}\right) \exp (i \omega t) d t\right]
$$

The electron-photon interaction operator $\Delta$ is proportional to $z$ for the linear polarization parallel to the $z$-axis in the electric dipole approximation. The reducible polarization $\pi$ is given in terms of irreducible polarization propagator $P$ and the screened Coulomb interaction $W\left(1=\left(x_{1}, t_{1}\right), x_{1}=\left(\mathbf{r}_{1}, \sigma_{1}\right)\right)$,

$$
\pi^{>}(1,2)=P^{>}(1,2)+\int_{c} d 3 d 4[P(1,3) W(3,4) P(4,2)]^{>}
$$

The integrals along the Keldysh contour are denoted as $\int_{c} \ldots$ The lowest order approximation in the skeleton expansion gives an explicit expression [28,29]

$$
i \pi^{>}\left(x t, x^{\prime}\right) \approx i P^{>}\left(x t, x^{\prime}\right) \approx g^{>}\left(x t, x^{\prime}\right) g^{<}\left(x^{\prime}, x t\right) .
$$

The greater electron Green's function $g^{>}$describes the propagation of excited photoelectrons, and the lesser electron Green's function $g^{<}$describes the hole propagation. We should notice that both $g^{>}$and $g^{<}$already include some correlation effects and phonon effects. In the core excitation processes the latter is well approximated by

$$
i g^{<}\left(x^{\prime}, x t\right)=-<\psi^{\dagger}(x t) \psi\left(x^{\prime}\right)>\approx-<\phi_{c}^{*}(x) \phi_{c}\left(x^{\prime}\right) b^{\dagger}(t) b>,
$$

where $b\left(b^{\dagger}\right)$ is the annihilation (creation) operator of the core state $\phi_{c}$. The average in the above equation is taken over electronic and phonon states. From now on we use the Born-Oppenheimer approximation (BOA) for simplicity. The core function $\phi_{c}$ is well approximated 


$$
\begin{aligned}
& \phi_{c}(x)=\phi_{c}\left(\mathbf{r}-\mathbf{R}_{A}^{0}-\mathbf{u}_{A}\right) \chi(\sigma), \\
& \phi_{c}(\mathbf{r})=R_{l_{c}}(r) Y_{L_{c}}(\hat{\mathbf{r}}) .
\end{aligned}
$$

As shown in the Appendix, for the small deviation $\mathbf{u}_{A}$, the core function is approximately given by

$$
\phi_{c}\left(\mathbf{r}-\mathbf{R}_{A}^{0}-\mathbf{u}_{A}\right) \approx \sum_{L} \phi_{L}^{c}\left(\mathbf{r}-\mathbf{R}_{A}^{0}\right) J_{L L_{c}}\left(\mathbf{u}_{A}\right)
$$

In eq. $(2.4) b^{\dagger}(t)=\exp (i H t) b^{\dagger} \exp (-i H t)$ includes the total Hamiltonian $H$ which is given in the BOA

$$
H=H_{e}+H_{v i b}^{0}=H_{e}^{*}+H_{v i b}^{*}
$$

where $H_{e}$ and $H_{v i b}^{0}$ are electronic and vibrational Hamiltonians with no core hole, whereas $H_{e}^{*}$ and $H_{v i b}^{*}$ are those with the core hole on $\phi_{c}$. They are explicitly written for the states before the core excitation [20] in terms of manyelectron Hamiltonian $H_{v}$ for the valence electrons and the core level energy $\varepsilon_{c}$,

$$
\begin{aligned}
& H_{e}=H_{v}+\varepsilon_{c}, \\
& H_{v i b}^{0}=\sum_{\nu} \omega_{\nu}\left(b_{\nu}^{\dagger} b_{\nu}+1 / 2\right),
\end{aligned}
$$

where $b_{\nu}\left(b_{\nu}^{\dagger}\right)$ is the phonon destruction (creation) operator for the mode $\nu=(\mathbf{q}, j) ; j$ is a phonon branch, $\mathbf{q}$ is the crystal momentum. After the core excitation

$$
\begin{aligned}
& H_{e}^{*}=H_{v}+V_{c}, \\
& H_{v i b}^{*}=H_{v i b}^{0}+\sum_{\nu}\left(B_{\nu} b_{\nu}+B_{\nu}^{*} b_{\nu}^{\dagger}\right) .
\end{aligned}
$$

Here $V_{c}$ is the core-hole potential, and the second term of $H_{v i b}^{*}$ is related to the displacement of the equilibrium configuration after the core excitation. We thus have a useful expression for the hole propagator $g^{<}$from eqs. (2.4), (2.6) and (2.7)

$$
\begin{aligned}
& i g^{<}\left(\mathbf{r}^{\prime}, \mathbf{r} t\right)=-\sum_{L L^{\prime}} \phi_{L}^{c}\left(\mathbf{r}-\mathbf{R}_{A}^{0}\right)^{*} \phi_{L^{\prime}}^{c}\left(\mathbf{r}^{\prime}-\mathbf{R}_{A}^{0}\right) \\
& \times<J_{L L_{c}}^{*}\left(\mathbf{u}_{A}\right) J_{L^{\prime} L_{c}}\left(\mathbf{u}_{A}\right) \exp \left(i H_{v i b}^{0} t\right) \exp \left(-i H_{v i b}^{*} t\right)>_{v i b} \\
& \times<\exp \left(i H_{e} t\right) b^{\dagger} \exp \left(-i H_{e}^{*} t\right) b>_{e}
\end{aligned}
$$


where $\langle\ldots\rangle_{v i b}$ and $\langle\ldots\rangle_{e}$ are the average over phonon states and electronic states. For example we have for the former

$$
<X>_{v i b}=\operatorname{Tr}\left[\exp \left(-\beta H_{v i b}^{0}\right) X\right] / \operatorname{Tr}\left[\exp \left(-\beta H_{v i b}^{0}\right)\right] .
$$

The difference between the two vibrational Hamiltonian $H_{v i b}^{0}$ and $H_{v i b}^{*}$ can contribute to the Franck-Condon factor $[1,2]$. At first we neglect the difference, which yields the simple formula for the vibrational expectation value in eq. (2.10) by use of the relation (A.12)

$$
\begin{aligned}
& <J_{L L_{c}}^{*}\left(\mathbf{u}_{A}\right) J_{L^{\prime} L_{c}}\left(\mathbf{u}_{A}\right)>_{v i b} \\
& =<J_{L_{c} L}\left(\mathbf{u}_{A}\right) J_{L^{\prime} L_{c}}\left(\mathbf{u}_{A}\right)>_{v i b} \\
& =(-1)^{l^{\prime}+l_{c}} \int d \hat{\mathbf{r}} d \hat{\mathbf{r}}^{\prime} Y_{L_{c}}^{*}(\hat{\mathbf{r}}) Y_{L}(\hat{\mathbf{r}}) Y_{L^{\prime}}^{*}\left(\hat{\mathbf{r}}^{\prime}\right) Y_{L_{c}}\left(\hat{\mathbf{r}}^{\prime}\right) \\
& \times<\exp \left[a \mathbf{u}_{A} \cdot\left(\hat{\mathbf{r}}^{\prime}-\hat{\mathbf{r}}\right)\right]>_{v i b} .
\end{aligned}
$$

For the phonon systems we apply the simplest approximation, the harmonic approximation. We can thus apply the Mermin's theorem [30] for the average in eq. (2.12)

$$
\begin{aligned}
& <\exp \left[a \mathbf{u}_{A} \cdot\left(\hat{\mathbf{r}}-\hat{\mathbf{r}}^{\prime}\right)\right]>_{v i b} \\
& =\exp \left[\frac{a^{2}}{2}<\left(\mathbf{u}_{A} \cdot\left(\hat{\mathbf{r}}-\hat{\mathbf{r}}^{\prime}\right)\right)^{2}>_{v i b}\right] .
\end{aligned}
$$

The average $<\left(\mathbf{u}_{A} \cdot\left(\hat{\mathbf{r}}-\hat{\mathbf{r}}^{\prime}\right)\right)^{2}>_{v i b}$ is simplified for the isotropic systems

$$
<\left(\mathbf{u}_{A} \cdot\left(\hat{\mathbf{r}}-\hat{\mathbf{r}}^{\prime}\right)\right)^{2}>_{v i b}=2 \sigma_{A}^{2}(1-\cos \theta)
$$

where we have used the relations

$$
\begin{aligned}
& <\left(\mathbf{u}_{A} \cdot \hat{\mathbf{r}}\right)^{2}>_{v i b}=<\left(\mathbf{u}_{A} \cdot \hat{\mathbf{r}}^{\prime}\right)^{2}>_{v i b}=<\mathbf{u}_{A}^{2}>_{v i b} / 3=\sigma_{A}^{2}, \\
& <\left(\mathbf{u}_{A} \cdot \hat{\mathbf{r}}\right)\left(\mathbf{u}_{A} \cdot \hat{\mathbf{r}}^{\prime}\right)>_{v i b}=\sigma_{A}^{2} \cos \theta,
\end{aligned}
$$

with $\cos \theta=\hat{\mathbf{r}} \cdot \hat{\mathbf{r}}^{\prime}$. From eqs. (2.13)-(2.15), the average given by eq. (2.12) is analytically given by

$$
\begin{aligned}
& <J_{L L_{c}}^{*}\left(\mathbf{u}_{A}\right) J_{L^{\prime} L_{c}}\left(\mathbf{u}_{A}\right)>_{v i b} \\
& =4 \pi(-1)^{l^{\prime}+l_{c}} \exp \left(a^{2} \sigma_{A}^{2}\right) \sum_{L_{1}} i^{l_{1}} j_{l_{1}}\left(i a^{2} \sigma_{A}^{2}\right) G\left(L_{c} L_{1} \mid L\right) G\left(L_{c} L_{1} \mid L^{\prime}\right)
\end{aligned}
$$

The most important term in the above sum arises from the term with $l_{1}=0$ and the next from those with $l_{1}=1$, because $j_{0}(i x)=\sinh (x) / x$ and $j_{l}(i x)$ 
are represented in the region where $x=a^{2} \sigma_{A}^{2} \ll 1$

$$
j_{l}(i x) \approx(i x)^{l} /(2 l+1) ! !,(l \geq 1) .
$$

We thus have an interesting formula for the deep core excitation from $\phi_{L_{c}}$ where atomic thermal motions are taken into account

$$
\begin{aligned}
& i g^{<}\left(x^{\prime}, x t\right) \approx\left[-\phi_{L_{c}}^{*}\left(\mathbf{r}-\mathbf{R}_{A}^{0}\right) \phi_{L_{c}}\left(\mathbf{r}^{\prime}-\mathbf{R}_{A}^{0}\right) \exp \left(a^{2} \sigma_{A}^{2}\right) j_{0}\left(i a^{2} \sigma_{A}^{2}\right)\right. \\
& +4 \pi i \sum_{L L^{\prime}} \phi_{L}^{c *}\left(\mathbf{r}-\mathbf{R}_{A}^{0}\right) \phi_{L^{\prime}}^{c}\left(\mathbf{r}^{\prime}-\mathbf{R}_{A}^{0}\right) \exp \left(a^{2} \sigma_{A}^{2}\right) j_{1}\left(i a^{2} \sigma_{A}^{2}\right) \\
& \left.\times \sum_{m_{1}} G\left(L_{c} 1 m_{1} \mid L\right) G\left(L_{c} 1 m_{1} \mid L^{\prime}\right)+\cdots\right]<\exp \left(i H_{e} t\right) b^{\dagger} \exp \left(-i H_{e}^{*} t\right) b>_{e} .
\end{aligned}
$$

In the second term, the selection rule for the Gaunt integral allows to pick up only the terms with $l, l^{\prime}=l_{c} \pm 1$. We thus have finite contribution from $p$-excitation even for the K-edge excitation because of the thermal motion of nuclei.

The second term in the large parenthesis of eq. (2.18) is simply given for the K-edge excitation $\left(l_{c}=0\right)$,

$$
\begin{aligned}
& \sum_{m_{1}} \phi_{1 m_{1}}^{c *}\left(\mathbf{r}-\mathbf{R}_{A}^{0}\right) \phi_{1 m_{1}}^{c}\left(\mathbf{r}^{\prime}-\mathbf{R}_{A}^{0}\right) f(\gamma) \\
& f(\gamma)=\exp (\gamma)(\gamma \cosh \gamma-\sinh \gamma) / \gamma^{2} \\
& \gamma=a^{2} \sigma_{A}^{2}
\end{aligned}
$$

For the $2 p$ excitation the large parenthesis of eq. (2.18) is given by a more complicated formula

$$
f(\gamma)\left[\phi_{00}^{c} \phi_{00}^{c}+\delta_{m_{c}, 0} A_{0}\left(\mathbf{r}, \mathbf{r}^{\prime}\right)+\delta_{m_{c}, \pm 1} A_{ \pm 1}\left(\mathbf{r}, \mathbf{r}^{\prime}\right)\right]
$$

where $A_{0}$ and $A_{ \pm 1}$ are explicitly given in terms of $\phi_{L}^{c}$

$$
\begin{aligned}
A_{0}= & \frac{2}{\sqrt{5}}\left[\phi_{00}^{c} \phi_{20}^{c}+\phi_{20}^{c} \phi_{00}^{c}\right]+\frac{3}{5}\left[\phi_{21}^{c *} \phi_{21}^{c}+\phi_{2,-1}^{c *} \phi_{2,-1}^{c}\right] \\
+ & \frac{4}{5} \phi_{20}^{c} \phi_{20}^{c}, \\
A_{ \pm 1}= & -\frac{1}{\sqrt{5}}\left[\phi_{00}^{c} \phi_{20}^{c}+\phi_{20}^{c} \phi_{00}^{c}\right] \\
& +\frac{1}{5}\left[\phi_{20}^{c} \phi_{20}^{c}+3 \phi_{2, \pm 1}^{c *} \phi_{2, \pm 1}^{c}+6 \phi_{2, \pm 2}^{c *} \phi_{2, \pm 2}^{c}\right] .
\end{aligned}
$$


We use the simplified notation as $\phi_{L}^{c} \phi_{L^{\prime}}^{c}=\phi_{L}^{c}\left(\mathbf{r}-\mathbf{R}_{A}^{0}\right) \phi_{L^{\prime}}^{c}\left(\mathbf{r}^{\prime}-\mathbf{R}_{A}^{0}\right)$.

The electronic part in eq. $(2.10)<\exp \left(i H_{e} t\right) b^{\dagger} \exp \left(-i H_{e}^{*} t\right) b>_{e}$ is reduced to the core spectral function $A_{c}^{*}$ whose complex conjugate is defined by $[20,28]$

$$
\begin{aligned}
& A_{c}(t)=<b^{\dagger} \exp \left(i H_{e}^{*} t\right) b \exp \left(-i H_{e} t\right)>_{e}=\sum_{n}\left|S_{n}\right|^{2} \exp \left(-i \varepsilon_{n} t\right), \\
& A_{c}(\omega)=\sum_{n}\left|S_{n}\right|^{2} \delta\left(\omega-\varepsilon_{n}\right), \\
& S_{n}=<n^{*}|b| 0>, \quad \varepsilon_{n}=E_{0}-E_{n}^{*}, \quad \sum_{n}\left|S_{n}\right|^{2}=1,
\end{aligned}
$$

where $\mid n^{*}>$ is the $n$th core-hole state which satisfies the equation (see eq. $(2.9))$

$$
H_{e}^{*}\left|n^{*}>=E_{n}^{*}\right| n^{*}>.
$$

The $n$th intrinsic loss probability is given by $\left|S_{n}\right|^{2}$. The extrinsic loss can be taken into account if we go beyond the lowest order skeleton expansion in eq. (2.2) [28]. In the present paper we will not discuss it any more.

\subsection{Pre-edge Structures}

So far we have focussed on the hole propagator $g^{<}$. Here we discuss the photoelectron or excited electron propagation. We thus discuss the particle propagator $g^{>}$. A useful expression of $g^{>}$is given in terms of particle Dyson orbital $f_{q}$

$$
\begin{aligned}
i g^{>}\left(x t, x^{\prime}\right) & =\sum_{q} f_{q}(x) f_{q}^{*}\left(x^{\prime}\right) \exp \left(-i \varepsilon_{q} t\right), \\
f_{q}(x) & =<0, N|\psi(x)| q, N+1> \\
\varepsilon_{q} & =E_{q}(N+1)-E_{0}(N)
\end{aligned}
$$

In one-electron approximation, $f_{q}(x)$ is simplified to a bound excited orbital or a continuum photoelectron wave function. Substituting eqs.(2.23)-(2.25), (2.3) in eq. (2.1), we obtain a formula to describe the pre-edge structures in K-edge X-ray absorption spectra where Franck-Condon effects are neglected but core displacement effects are considered,

$$
\begin{aligned}
I\left(\omega_{k}\right) & =2 \pi \sum_{q}\left|<f_{q}\right| \Delta\left|\phi_{1 s}>\right|^{2} f_{0}(\gamma) A_{c}\left(\varepsilon_{q}-\omega_{k}\right) \\
& +2 \pi \sum_{q m}\left|<f_{q}\right| \Delta\left|\phi_{1 m}^{1 s}>\right|^{2} f(\gamma) A_{c}\left(\varepsilon_{q}-\omega_{k}\right),
\end{aligned}
$$




$$
f_{0}(\gamma)=\exp (\gamma) \sinh (\gamma) / \gamma
$$

The first term describes the conventional X-ray absorption intensity for $1 s \rightarrow$ $f_{q}$ transition, which has phonon effects in the factor $f_{0}(\gamma)$. The second describes the absorption intensity induced by atomic displacement due to the thermal motion. An explicit expression of the electron-photon interaction operator $\Delta$ is now given neglecting unimportant numerical factor

$$
\begin{gathered}
\Delta=\mathbf{e} \cdot \mathbf{r}+\frac{i}{2}(\mathbf{e} \cdot \mathbf{r})(\mathbf{k} \cdot \mathbf{r}), \\
+\frac{1}{2 \omega_{k}}(\mathbf{k} \times \mathbf{e}) \cdot \mathbf{L} .
\end{gathered}
$$

The first term describes the electric dipole (E1), the second the electric quadrupole (E2), and the third the magnetic dipole (M1) transitions. In the above formula, $\mathbf{e}$ and $\mathbf{k}$ are the photon polarization and the propagation vectors; we notice that $|\mathbf{e}|=1, \omega_{k}=c k$, and $\mathbf{e} \cdot \mathbf{k}=0$. We should note that both of the first and the second terms in eq. (2.26) depend on temperature through $f_{0}(\gamma)$ and $f(\gamma)$ given by eq. (2.20).

A lowest order approximation for $f_{q}(x)$ to discuss pre-edge structures in X-ray absorption spectra is thus given by

$$
f_{q}(x) \approx \phi_{q}(x)
$$

where $\phi_{q}(x)$ is localized atomic orbital on the X-ray absorbing atom $A$. Even though the function is localized, the "size" of the function $\phi_{q}$ is much larger than the atomic thermal fluctuation. In contrast the deep core function $\phi_{c}$ has non-negligible effects from the thermal motion. Now we consider $1 s \rightarrow 3 d$ transitions in transition metals, where they have vacant $3 d$ levels on each composite atom. As far as we discuss the transitions to the vacant quasilocalized $3 d$ levels, only $1 s \rightarrow 3 d$ E2 transitions ( first term in eq. (2.26)) are allowed in the static approximation. In addition we take the atomic thermal motion into account, then the E1 transition $\phi_{1 m}^{1 s} \rightarrow 3 d$ (2nd term in eq.(2.26)) is also allowed.

Now let calculate the temperature dependence of the pre-edge intensity where the incident linearly polarized X-ray $\| z$ is propagating along $x$ direction. The thermal factor $\sigma_{A}$ is the same as that used in X-ray diffraction analyses. We use the harmonic approximation for the phonon modes, and in the temperature range $100 K<T$ the classical approximation is assumed to work well. We thus use a simple expression for the thermal factor

$$
\sigma_{A}^{2}=\sigma_{0}^{2} \frac{T}{300}
$$


where $\sigma_{0}^{2}$ is the thermal factor at $300 \mathrm{~K}$. The Ti $1 s$ exponent $a$ is $21.44,3 d$ exponent is 2.71. Figures 1 (a-d) show the temperature dependence of the pre-edge intensity for Ti K-edge excitation. In this geometrical setup $1 s \rightarrow 3 d_{ \pm 1}$ transitions are allowed in the E2 transitions induced by the thermal displacement, whereas $\phi_{1 m}^{1 s} \rightarrow 3 d_{ \pm 1}, 3 d_{0}$ are allowed in the E1 transition because of the thermal atomic vibrations. We calculate the temperature dependences for four different $\sigma_{0}^{2}$; from $10^{-2}$ to $10^{-5}$ a.u. ${ }^{2}$. For small $\sigma_{A}$, we have the temperature dependence in linear function of $T$, however the nonlinear effect is prominent for the large temperature factor $\sigma_{0}^{2}=0.01 a . u .^{2}$; there the E1 intensity caused by the thermal vibration is much stronger than the E2 transition. Even for the value $\sigma_{0}^{2}=0.0001 a . u .^{2}$, the E1 intensity is still stronger than the E2 absorption intensity. For the small value $\sigma_{0}^{2}=10^{-5} a . u .^{2}$, they are in the same order. These behaviors are similar to those observed in Ti K-edge XANES (pre-edge ) spectra for $\mathrm{SrTiO}_{3}[5]$.

\subsection{XANES Spectra}

For the XANES analyses, the detailed calculations of $f_{q}$ 's are too complicated and impractical for condensed systems because they extend over the whole systems. We again use the formula for the hole propagator $g^{<}$in terms of $\phi_{1 s}$ and $\phi_{1 m}^{1 s}$ for the K-edge excitation, and $\phi_{2 p_{m}}, \phi_{00}^{2 p}$ and $\phi_{2 m}^{2 p}$ for the $\mathrm{L}_{23}$-edge excitation, but we use $g^{>}$for the photoelectron propagation, which yields a XANES formula for the K-edge excitation,

$$
\begin{aligned}
I\left(\omega_{k}\right)= & -\frac{1}{2 \pi} \operatorname{Im}\left[<\phi_{1 s}\left|\Delta^{*} g^{>}\left(\varepsilon_{0}+\omega_{k}\right) \Delta\right| \phi_{1 s}>f_{0}(\gamma)\right. \\
& \left.+\sum_{m_{1}}<\phi_{m_{1}}^{1 s}\left|\Delta^{*} g^{>}\left(\varepsilon_{0}+\omega_{k}\right) \Delta\right| \phi_{1 m_{1}}^{1 s}>f(\gamma)\right] .
\end{aligned}
$$

In the above equation $\operatorname{Im}\left[<\phi\left|\Delta^{*} g^{>}\left(\varepsilon_{0}+\omega_{k}\right) \Delta\right| \phi\right\rangle$ is rewritten in terms of the retarded Green's function $g^{r}$ instead of $g^{>}$[28] and the XANES formula is now reduced to

$$
\begin{aligned}
I\left(\omega_{k}\right)= & -\frac{1}{\pi} \operatorname{Im}\left[<\phi_{1 s}\left|\Delta^{*} g^{r}\left(\varepsilon_{0}+\omega_{k}\right) \Delta\right| \phi_{1 s}>f_{0}(\gamma)\right. \\
& \left.+\sum_{m_{1}}<\phi_{m_{1}}^{1 s}\left|\Delta^{*} g^{r}\left(\varepsilon_{0}+\omega_{k}\right) \Delta\right| \phi_{1 m_{1}}^{1 s}>f(\gamma)\right] .
\end{aligned}
$$

The retarded Green's function $g^{r}$ plays the same role as the scattering Green's function because of the same boundary condition. We can thus apply the multiple scattering theory to both the first and the second terms in the above equation.

For the description of photoelectron propagation, Debye-Waller factors play 
an important role in particular EXAFS region. Even in the XANES region the Debye-Waller factors are essential to study the temperature dependence: We have to take the spherical wave effects. XANES Debye-Waller factors have been discussed before [32], however we give here a different practical formula to clarify the mathematical structure of the matrix $J$ discussed in the appendix A. The XANES formula (2.32) is conveniently calculated by use of renormalized multiple scattering series. • • Both the first and the second terms in eq. (2.32) are written in the form within the muffin-tin approximation

$$
\begin{aligned}
& -\frac{8}{3} \operatorname{Im} \sum_{L L^{\prime}} i^{l-l^{\prime}} \exp \left[i\left(\delta_{l}^{A}+\delta_{l^{\prime}}^{A}\right)\right] \rho_{c}(l) \rho_{c}\left(l^{\prime}\right) G\left(L_{c} 10 \mid L\right) G\left(L_{c} 10 \mid L^{\prime}\right) \\
& \times\left[(t-X t)^{-1}\right]_{L L^{\prime}}^{A A}
\end{aligned}
$$

where $t_{L L^{\prime}}^{\alpha \beta}=\delta^{\alpha \beta} \delta_{L L^{\prime}} t_{l}^{\alpha}$ and $X$ is given by

$$
\begin{aligned}
& X_{L L^{\prime}}^{\alpha \beta}=t_{l}^{\alpha}(k) G_{L L^{\prime}}\left(k \mathbf{R}_{\alpha}-k \mathbf{R}_{\beta}\right)\left(1-\delta^{\alpha \beta}\right), \\
& t_{l}^{\alpha}(k)=-\left[\exp \left(2 i \delta_{l}^{\alpha}\right)-1\right] /(2 i k) .
\end{aligned}
$$

The propagator $G_{L L^{\prime}}\left(k \mathbf{R}_{\alpha}-k \mathbf{R}_{\beta}\right)$ describes the photoelectron propagation from the site $\beta$ with orbital angular momentum $L^{\prime}$ to $\alpha$ with $L$. The radial matrix element $\rho_{c}(l)=\int d r R_{l}(k r) R_{l_{c}}(r) r^{3}$ in eq. (2.33) is calculated by use of $R_{1 s}$, and for the second by use of $R_{1}^{1 s}$ for $R_{l_{c}}$.

For small displacement $\mathbf{u}$, the propagator $G_{L L^{\prime}}\left(k \mathbf{R}^{0}+k \mathbf{u}\right)$ is related to $G_{L L^{\prime}}\left(k \mathbf{R}^{0}\right)$ by use of the matrix $\hat{J}[33]$

$$
\begin{aligned}
& G_{L L^{\prime}}\left(k \mathbf{R}^{0}+k \mathbf{u}\right)=\sum_{L_{1}} G_{L L_{1}}\left(k \mathbf{R}^{0}\right) \hat{J}_{L_{1} L^{\prime}}(k \mathbf{u}), \\
& \hat{J}_{L_{1} L^{\prime}}(k \mathbf{u})=\int d \hat{\mathbf{r}} Y_{L_{1}}^{*}(\hat{\mathbf{r}}) \exp (i k \mathbf{u} \cdot \hat{\mathbf{r}}) Y_{L^{\prime}}(\hat{\mathbf{r}}) .
\end{aligned}
$$

We should note that the matrix $\hat{J}$ is obtained from $J$ defined by eq. (A.12) applying the analytical continuation $a \rightarrow-i k$. Let us consider a double scattering X-ray absorption formula for the $A \rightarrow \alpha \rightarrow \beta \rightarrow A$ path with atomic displacement, which has a matrix product of $\hat{J}$ 's

$$
\sum_{L_{1} L_{1}^{\prime} L_{2} L_{2}^{\prime} L_{3}} G_{L L_{1}}\left(k \mathbf{R}_{A \beta}\right) \hat{J}_{L_{1} L_{1}^{\prime}}\left(k \mathbf{u}_{A \beta}\right) X_{L_{1}^{\prime} L_{2}}^{\beta \alpha} \hat{J}_{L_{2} L_{2}^{\prime}}\left(k \mathbf{u}_{\beta \alpha}\right) X_{L_{2}^{\prime} L_{3}}^{\alpha A} \hat{J}_{L_{3} L^{\prime}}\left(k \mathbf{u}_{\alpha A}\right) .
$$

The thermal average of the above formula for the harmonic phonon systems yields with aid of the Mermin's theorem [30]

$$
<\hat{J}_{L_{1} L_{1}^{\prime}}\left(k \mathbf{u}_{A \beta}\right) \hat{J}_{L_{2} L_{2}^{\prime}}\left(k \mathbf{u}_{\beta \alpha}\right) \hat{J}_{L_{3} L^{\prime}}\left(k \mathbf{u}_{\alpha A}>\right.
$$




$$
\begin{aligned}
& =\int d \hat{\mathbf{r}_{1}} \hat{\mathbf{r}}_{2} d \hat{\mathbf{r}}_{3} Y_{L_{1}}^{*}\left(\hat{\mathbf{r}}_{1}\right) Y_{L_{1}^{\prime}}\left(\hat{\mathbf{r}}_{1}\right) Y_{L_{2}}^{*}\left(\hat{\mathbf{r}}_{2}\right) Y_{L_{2}^{\prime}}\left(\hat{\mathbf{r}}_{2}\right) Y_{L_{3}}^{*}\left(\hat{\mathbf{r}}_{3}\right) Y_{L^{\prime}}\left(\hat{\mathbf{r}}_{3}\right) \\
& \times<\exp \left(i k \mathbf{u}_{A \beta} \cdot \hat{\mathbf{r}}_{1}\right) \exp \left(i k \mathbf{u}_{\beta \alpha} \cdot \hat{\mathbf{r}}_{2}\right) \exp \left(i k \mathbf{u}_{\alpha A} \cdot \hat{\mathbf{r}}_{3}\right)> \\
& =\int d \hat{\mathbf{r}_{1}} \hat{\mathbf{r}}_{2} d \hat{\mathbf{r}}_{3} Y_{L_{1}}^{*}\left(\hat{\mathbf{r}}_{1}\right) Y_{L_{1}^{\prime}}\left(\hat{\mathbf{r}}_{1}\right) Y_{L_{2}}^{*}\left(\hat{\mathbf{r}}_{2}\right) Y_{L_{2}^{\prime}}\left(\hat{\mathbf{r}}_{2}\right) Y_{L_{3}}^{*}\left(\hat{\mathbf{r}}_{3}\right) Y_{L^{\prime}}\left(\hat{\mathbf{r}}_{3}\right) \\
& \times \exp \left[-\frac{k^{2}}{2}\left(<\left(\mathbf{u}_{A \beta} \cdot \hat{\mathbf{r}}_{1}\right)^{2}>+\ldots+<\left(\mathbf{u}_{\alpha A} \cdot \hat{\mathbf{r}}_{3}\right)^{2}>\right)\right] \\
& \times \exp \left[-\frac{k^{2}}{2}\left(<\left(\mathbf{u}_{A \beta} \cdot \hat{\mathbf{r}}_{1}\right)\left(\mathbf{u}_{\beta \alpha} \cdot \hat{\mathbf{r}}_{2}\right)>+\ldots\right)\right] .
\end{aligned}
$$

In the complicated multiple scattering paths the cross terms as shown in the last exponential term are expected to be canceled out because of their random phases. By use of this assumption we can define the temperature dependent path matrix $X(T)$ instead of the temperature independent $X$ defined by eq. $(2.34)$

$$
\begin{aligned}
& X_{L L^{\prime}}^{\beta \alpha}(T)=\sum_{L^{\prime \prime}} X_{L L^{\prime \prime}}^{\beta \alpha} K_{L^{\prime \prime} L^{\prime}}^{\beta \alpha}(T), \\
& K_{L^{\prime \prime} L^{\prime}}^{\beta \alpha}(T)=\int d \hat{\mathbf{r}} Y_{L^{\prime \prime}}^{*}(\hat{\mathbf{r}}) \exp \left[-\frac{k^{2}}{2}<\left(\mathbf{u}_{\beta \alpha} \cdot \hat{\mathbf{r}}\right)^{2}>\right] Y_{L^{\prime}}(\hat{\mathbf{r}}) .
\end{aligned}
$$

For the isotropic systems we can simplify the matrix $K$ and $X(T)$

$$
X_{L L^{\prime}}^{\beta \alpha}(T)=X_{L L^{\prime}}^{\beta \alpha} \exp \left[-\frac{k^{2}}{6}<\mathbf{u}_{\beta \alpha}^{2}>\right]
$$

The thermal factor $\left\langle\mathbf{u}_{\beta \alpha}^{2}\right\rangle$ is related to the conventional EXAFS thermal factor

$$
\begin{aligned}
& <\left(\hat{\mathbf{R}} \cdot\left(\mathbf{u}_{\beta}-\mathbf{u}_{\alpha}\right)\right)^{2}>=\Delta_{\beta \alpha}^{2} \\
& <u_{\beta \alpha}^{2}>=3 \Delta_{\beta \alpha}^{2}
\end{aligned}
$$

For the systems with axial symmetry around $z$-axis the matrix $K(T)$ is more complicated and read

$$
K_{L^{\prime \prime} L^{\prime}}^{\beta \alpha}(T)=\exp \left[-\frac{k^{2}}{6}<\mathbf{u}_{\beta \alpha}^{2}>\right]\left[\delta_{L^{\prime \prime} L^{\prime}}-\frac{k^{2}}{3} \Delta \sigma_{\beta \alpha}^{2} G\left(L^{\prime} 20 \mid L^{\prime \prime}\right)+\ldots\right]
$$

where

$$
\Delta \sigma_{\beta \alpha}^{2}=<u_{\beta \alpha, z}^{2}>-<u_{\beta \alpha, x}^{2}>
$$




\subsection{Franck-Condon factor}

So far we have neglected the difference of the vibrational Hamiltonian for the no-hole electronic states $H_{v i b}^{0}$ and that for the core-hole states $H_{v i b}^{*}$ in eq. (2.10). Now we study the influence of the difference responsible for the Franck-Condon effects. By applying Schwinger's technique [34,35] we have

$$
\begin{aligned}
& \exp \left(i H_{v i b}^{0} t\right) \exp \left(-i H_{v i b}^{*} t\right)=\exp [-i C(t)+B(t)] \\
& B(t)=\sum_{\nu}\left[g_{\nu}(t) b_{\nu}-g_{\nu}^{*}(t) b_{\nu}^{\dagger}\right] \\
& C(t)=\sum_{\nu} \frac{\left|\lambda_{\nu}\right|^{2}}{\omega_{\nu}}\left(t-\frac{\sin \omega_{\nu} t}{\omega_{\nu}}\right) \\
& g_{\nu}(t)=\lambda_{\nu} \frac{\exp \left(-i \omega_{\nu} t\right)-1}{\omega_{\nu}} .
\end{aligned}
$$

The factor $C(t)$ is not a q-number but a c-number. Substitution of eq. (2.43) into eq. (2.10) in the average over the vibrational states yields a more complicated formula than eq. (2.16)

$$
\begin{aligned}
& <J_{L L_{c}}^{*}\left(\mathbf{u}_{A}\right) J_{L^{\prime} L_{c}}\left(\mathbf{u}_{A}\right) \exp \left(i H_{v i b}^{0} t\right) \exp \left(-i H_{v i b}^{*} t\right)>_{v i b} \\
& =(-1)^{l^{\prime}+l_{c}} \int d \hat{\mathbf{r}} d \hat{\mathbf{r}}^{\prime} Y_{L_{c}}^{*}(\hat{\mathbf{r}}) Y_{L}(\hat{\mathbf{r}}) Y_{L^{\prime}}^{*}\left(\hat{\mathbf{r}}^{\prime}\right) Y_{L_{c}}\left(\hat{\mathbf{r}}^{\prime}\right) \\
& \times<\exp \left[a \mathbf{u}_{A} \cdot\left(\hat{\mathbf{r}}^{\prime}-\hat{\mathbf{r}}\right)\right] \exp [B(t)]>_{v i b} \exp [-i C(t)]
\end{aligned}
$$

Again we apply the Mermin's theorem [30]; the average over the phonon states $\langle\ldots\rangle_{v i b}$ is then given with aid of the isotropic and harmonic approximation by

$$
\exp \left[a^{2} \sigma_{A}^{2}(1-\cos \theta)+a<\mathbf{u}_{A} \cdot\left(\hat{\mathbf{r}}^{\prime}-\hat{\mathbf{r}}\right) B(t)>+\frac{1}{2}<B^{2}(t)>\right]
$$

The first term is just due to the thermal displacement of absorbing atom A, the third is the Franck-Condon factor and the second is their interference term, which is explicitly given by

$$
\mathbf{a}(t) \cdot\left(\hat{\mathbf{r}}^{\prime}-\hat{\mathbf{r}}\right),
$$

where we have defined the vector $\mathbf{a}(t)$

$$
\mathbf{a}(t)=\sum_{\nu} \frac{a}{\sqrt{2 N M_{A} \omega_{\nu}}}\left[\mathbf{e}^{*}(\nu) g_{\nu}(t) n\left(\omega_{\nu}\right)-\mathbf{e}(\nu) g_{\nu}^{*}(t)\left(n\left(\omega_{\nu}\right)+1\right)\right]
$$


where $M_{A}$ is the mass of the X-ray absorbing atom $A, n(\omega)$ is the average phonon number $n(\omega)=[\exp (\beta \omega)-1]^{-1}, \mathbf{e}(\nu)$ is the phonon polarization vector for $\nu=(\mathbf{q}, j)$ (crystal momentum $\mathbf{q}$, branch $j$ ). The first order contribution from the interference to the average (2.46) should vanish because of the symmetry. The Franck-Condon factor $\left\langle B^{2}(t)>/ 2\right.$ is easily calculated in the harmonic vibration approximation $[15,20]$, and is given by

$$
\begin{aligned}
& -i C(t)+\frac{1}{2}<B^{2}(t)>=-\alpha+i \beta t \\
& +\int_{0}^{\infty} d \omega \frac{D(\omega)}{\omega^{2}}[(n(\omega)+1) \exp (-i \omega t)+n(\omega) \exp (i \omega t)]
\end{aligned}
$$

where

$$
\begin{aligned}
D(\omega) & =\sum_{\nu}\left|\lambda_{\nu}\right|^{2} \delta\left(\omega-\omega_{\nu}\right), \\
\alpha & =\int_{0}^{\infty} d \omega \frac{D(\omega)}{\omega^{2}}[2 n(\omega)+1], \\
\beta & =\int_{0}^{\infty} d \omega \frac{D(\omega)}{\omega} .
\end{aligned}
$$

Following Almbladh and Hedin [20], we define the spectral function

$$
F(t)=\exp \left[-i C(t)+\frac{1}{2}<B^{2}(t)>\right]=\int_{-\infty}^{\infty} F(\omega) \exp (-i \omega t) d \omega
$$

which satisfies the normalization condition

$$
\int F(\omega) d \omega=F(t=0)=1 .
$$

We thus have

$$
\begin{aligned}
& <\omega>=\int \omega F(\omega) d \omega=i \dot{F}(t=0)=0, \\
& <\omega^{2}>=-\ddot{F}(t=0)=\int_{0}^{\infty} d \omega D(\omega)[2 n(\omega)+1] .
\end{aligned}
$$

The Franck-Condon broadening increases as a function of temperature $T$. We thus understand the Franck-Condon and also its interference with the core displacement have no influence on the peak shift. The factor $\exp (-\alpha)$ where 
$\alpha$ is given by eq. (2.50) has a finite contribution to the thermal damping due to the Franck-Condon effects contrary to the core displacement.

\section{5 quasi-particle energy for the core state}

The quasi-particle energy (binding energy) of the core level $\varepsilon_{c}=E_{0}-E_{c}^{*}$ defined by eq. (2.24) is calculated by use of a pole of the retarded Green's function. We keep only the diagonal part of the electron selfenergy with respect to the core function $\phi_{c}$,

$$
\varepsilon_{c}=<c\left|h+V_{H}\right| c>+<c\left|\Sigma^{r}\left(\varepsilon_{c}\right)\right| c>
$$

where $h$ is one-electron part and $V_{H}$ is the temperature dependent Hartree potential

$$
\begin{aligned}
V_{H}(\mathbf{r}) & =\int d \mathbf{r}^{\prime} v\left(\mathbf{r}-\mathbf{r}^{\prime}\right)<\rho\left(\mathbf{r}^{\prime}\right)> \\
<\rho(\mathbf{r})> & =\sum_{\sigma}<\psi^{\dagger}(x) \psi(x)>.
\end{aligned}
$$

Of course $h+V_{H}$ directly depends on atomic displacement and also thermal electron excitations near Fermi level. These effects are however small compared with the core displacement effects on the core wave functions as discussed before. By use of the relations (2.6) and (2.16), we have an explicit formula within the temperature Hartree approximation for the K-edge excitation

$$
\begin{aligned}
& <c\left|h+V_{H}\right| c>_{v i b}=\varepsilon_{0}^{H} f_{0}(\gamma)+\sum_{m} \varepsilon_{m}^{H} f(\gamma), \\
& \varepsilon_{m}^{H}=<\phi_{1 m}\left|h+V_{H}\right| \phi_{1 m}>.
\end{aligned}
$$

We have used the conventional Hartree energy $\varepsilon_{0}^{H}$ without atomic displacement, typically negative, and $\varepsilon_{m}^{H}$ the Hartree energy induced by core displacement. Both $f_{0}(\gamma)$ and $f(\gamma)$ are increasing function of the temperature $T$.

We can obtain a similar formula as far as we neglect the atomic displacement effects on the selfenergy $\Sigma^{r}$,

$$
<c\left|h+V_{H}+\Sigma^{r}\left(\varepsilon_{c}\right)\right| c>=\varepsilon_{0} f_{0}(\gamma)+\sum_{m} \varepsilon_{m} f(\gamma)
$$

where $\varepsilon_{0}$ and $\varepsilon_{m}$ are quasi-particle energy without and with core displacement 


$$
\begin{aligned}
\varepsilon_{0} & =<\phi_{c}\left|h+V_{H}+\Sigma^{r}\left(\varepsilon_{c}\right)\right| \phi_{c}>, \\
\varepsilon_{m} & =<\phi_{1 m}\left|h+V_{H}+\Sigma^{r}\left(\varepsilon_{c}\right)\right| \phi_{1 m}>.
\end{aligned}
$$

We thus have the binding energy shift with temperature

$$
\Delta \varepsilon_{c}=-\varepsilon_{0}\left[f_{0}(\gamma)-1\right]-\sum_{m} \varepsilon_{m} f(\gamma)
$$

For the very small deviation limit, the binding energy shift behaves as

$$
\Delta \varepsilon_{c} \approx\left(\varepsilon_{0}+\frac{1}{3} \sum_{m} \varepsilon_{m}\right) \gamma
$$

If we neglect the sum $\sum_{m} \varepsilon_{m}$ induced by atomic displacement, negative $\varepsilon_{0}$ gives only the energy shift toward higher binding energy side. Recent experimental results by Mannel et al. [6], however, show the pre-edge shift toward lower energy for higher temperature, which suggests the finite contribution from the second term of eqs. (2.60) and (2.61).

Figure 2 shows the binding energy shifts $\Delta \varepsilon_{c}$ as a function of temperature for Ti K-edge excitation, where the ratio $\beta$ defined by

$$
\beta=\sum_{m} \varepsilon_{m} / \varepsilon_{0}
$$

is changed from -2.85 to -3.15 . For the ratios $-2.85,-2.90$ and -2.95 , the shift $\Delta \varepsilon_{c}$ increases as $T$, whereas it decreases for the smaller rations, $-3.00 \sim$ -3.15 . The direct evaluation of $\varepsilon_{m}$ is rather hard. So far no prominent binding energy shift has been observed, which suggests that $\varepsilon_{0} \approx-\varepsilon_{m}$.

\section{Core Photoemission}

In this section, we discuss phonon effects on photoemission from a deep core $\phi_{c}$ excited by X-ray photons. In the Keldysh Green's function approach to the photoemission theory $[24,36]$, the photoemission intensity without extrinsic losses and resonant processes is given in terms of dressed photoelectron function $f_{\mathrm{p}}^{-}$under the influence of the optical potential (advanced electron self-energy $\Sigma^{a}$ ) and $g^{<}$,

$$
I(\mathbf{p}) \propto \operatorname{Im}<f_{\mathbf{p}}^{-}\left|\Delta g^{<}\left(\varepsilon_{p}-\omega\right) \Delta^{*}\right| f_{\mathbf{p}}^{-}>
$$


where $g^{<}\left(\varepsilon_{p}-\omega\right)$ is Fourier-transformed lesser Green's function

$$
g^{<}\left(\varepsilon_{p}-\omega\right)=\int d t g^{<}(t) \exp \left[i\left(\varepsilon_{p}-\omega\right) t\right]
$$

The integral (3.1) is thus given by use of eq. (2.10)

$$
\begin{aligned}
& <f_{\mathbf{p}}^{-}\left|\Delta g^{<}(t) \Delta^{*}\right| f_{\mathbf{p}}^{-}> \\
& =i \sum_{L L^{\prime}}<f_{\mathbf{p}}^{-}|\Delta| \phi_{L^{\prime}}^{c}><\phi_{L}^{c}\left|\Delta^{*}\right| f_{\mathbf{p}}^{-}> \\
& \times<\exp \left(i H_{e} t\right) b^{\dagger} \exp \left(-i H_{e}^{*} t\right) b>_{e} \\
& \times<J_{L L_{c}}^{*}\left(\mathbf{u}_{A}\right) J_{L^{\prime} L_{c}}\left(\mathbf{u}_{A}\right) \exp \left(i H_{v i b}^{0} t\right) \exp \left(-i H_{v i b}^{*} t\right)>_{v i b} .
\end{aligned}
$$

At first we neglect the difference between the phonon Hamiltonian $H_{v i b}^{0}$ and $H_{v i b}^{*}$. In this approximation the right hand side of eq. (3.3) is written for the K-edge excitation,

$$
\begin{aligned}
& <f_{\mathbf{p}}^{-}\left|\Delta g^{<}(t) \Delta^{*}\right| f_{\mathbf{p}}^{-}> \\
& \approx\left[\left|<f_{\mathbf{p}}^{-}\right| \Delta\left|\phi_{00}^{c}>\right|^{2} f_{0}(\gamma)+\sum_{m}\left|<f_{\mathbf{p}}^{-}\right| \Delta\left|\phi_{1 m}^{c}>\right|^{2} f(\gamma)\right] \\
& \times<\exp \left(i H_{e} t\right) b^{\dagger} \exp \left(-i H_{e}^{*} t\right) b>_{e}
\end{aligned}
$$

where $f(\gamma)$ is given by the thermal factor as shown by eq. $(2.20)$, and $f_{0}(\gamma)$ is given by eq. (2.27). The first term in eq. (3.4) describes the photoemission processes where the core function is not polarized, but the second term describes those processes associated with the core polarization due to the atomic thermal displacement. The thermal fluctuation, however, influences the first term through $f_{0}(\gamma)$. The electric dipole transition yields $p$-wave in the first term, whereas $d$ - and $s$-waves in the second term at the excited atom because of the E1 transition. After the excitation the photoelectron waves propagate inside solids and suffer multiple scatterings from surrounding composite atoms. The last factor in eq. (3.4) is the same as $A_{c}^{*}(t)$ in eq. (2.23). We thus have a formula for the photoemission intensity $I(\mathbf{p})$ including the effects due to the thermal atomic displacement at K-edge excitation

$$
\begin{aligned}
& I(\mathbf{p}) \propto \sum_{n}\left|S_{n}\right|^{2}\left(\left|<f_{\mathbf{p}}^{-}\right| \Delta\left|\phi_{00}^{c}>\right|^{2} f_{0}(\gamma)\right. \\
& \left.+\sum_{m}\left|<f_{\mathbf{p}}^{-}\right| \Delta\left|\phi_{1 m}^{c}>\right|^{2} f(\gamma)\right) \delta\left(\varepsilon_{p}+E_{n}^{*}-E_{0}-\omega\right),
\end{aligned}
$$

where only the intrinsic loss effects are taken into account. The second term in the large parenthesis is due to the atomic displacement, which gives rise to quite different photoelectron angular distribution from the first term. As 
far as we completely neglect the elastic scatterings from nearby atoms, the latter (the first term) shows simply $\cos ^{2} \theta$ angular distribution, where $\theta$ is the angle between the X-ray polarization and the photoelectron momentum $\mathbf{p}$. • • For real systems, we cannot neglect the elastic scatterings as shown in the studies of plasmon losses in XPS spectra $[37,38]$. We rather show here the photoelectron angular distribution to demonstrate and to stress the influence of the thermal atomic displacement without elastic scatterings

Figure 3 (a) and (b) show the photoelectron angular distribution excited from Si K-edge at $\varepsilon_{p}=100 \mathrm{eV}$ : In (a) $\sigma_{0}^{2}=0.005 a . u .^{2}$ and 0.01a.u. ${ }^{2}$ in (b). For simplicity we neglect elastic scatterings from surrounding atoms. That is, only $A$ term in eq. (3.8) is taken into account. We normalize the intensity so that the integrated intensity over $\theta$ to be the same. We observe that the normalized intensity decreases at $\theta=0$, whereas increases at large $\theta>\approx 30^{\circ}$ with temperature. For the larger $\sigma_{0}$ the angular deviation from the $\cos ^{2} \theta$ is prominent as shown in Fig. 3 (b). Figure 4 (a) and (b) show the photoelectron angular distribution excited from Ti K-edge at $\varepsilon_{p}=100 \mathrm{eV}$ : In (a) $\sigma_{0}^{2}=0.005 a . u .^{2}$ and 0.01a.u. ${ }^{2}$ in (b). For simplicity we neglect elastic scatterings from surrounding atoms, and normalize the intensity so that the integrated intensity over $\theta$ to be the same. We observe that the normalized intensity decreases at $\theta=0$, whereas increases at large $\theta>\approx 30^{\circ}$ with temperature. For the larger $\sigma_{0}$ the angular deviation from the $\cos ^{2} \theta$ is prominent as shown in Fig. 4 (b). The comparison with the result for Si K-edge excitation demonstrates that for the heavier atom $\mathrm{Ti}$ the thermal deviation has prominent effects on the angular distribution, because the core size is much smaller for Ti than Si. Figure 5 shows the similar results for $\mathrm{Cu}$ K-edge excitation. Even for the smaller DW factors the prominent deviation from the $\cos ^{2} \theta$ angular dependence is observed because of the smaller size of $1 s$ core function of $\mathrm{Cu}$.

Next we study the influence of the difference between $H_{v i b}^{*}$ and $H_{v i b}^{0}$ in eq. (3.3) which contributes to the Franck-Condon effects. The thermal average in eq. (3.3) gives rise to the quite similar formula shown in subsection 2.4: The Franck-Condon and its interference with the core displacement have no influence on the peak shift, but finite effects on the peak broadening and the intensity damping.

In addition to this factor we should consider the Debye-Waller factors for polyatomic systems. The scattering effects from surrounding atoms are completely neglected so far. The interference effects (photoelectron diffraction effects) are suppressed because of the DW factors in particular in high-energy region. The photoemission amplitude is then written in terms of the damping plane wave $\phi_{\mathbf{p}}^{0}$, full T-matrix $T$ expanded in terms of site T-matrix $t_{\alpha}$, and damping free propagator $g_{0}(\varepsilon)=\left(\varepsilon+\nabla^{2} / 2+i \Gamma\right)^{-1}(\Gamma>0$ is the imaginary part of the optical potential ) $[11,39-41]$ 


$$
\begin{aligned}
& <f_{\mathbf{p}}^{-}|\Delta| \phi_{c}>=<\phi_{\mathbf{p}}^{0}\left|\left[1+T g_{0}\left(\varepsilon_{p}\right)\right] \Delta\right| \phi_{c}> \\
& =<\phi_{A \mathbf{p}}^{-}|\Delta| \phi_{c}>+\sum_{\alpha(\neq A)}<\phi_{\mathbf{p}}^{0}\left|t_{\alpha} g_{A} \Delta\right| \phi_{c}> \\
& +\sum_{\beta \neq \alpha(\neq A)}<\phi_{\mathbf{p}}^{0}\left|t_{\beta} g_{0} t_{\alpha} g_{A} \Delta\right| \phi_{c}>+\ldots, \\
& <\phi_{\mathbf{p}}^{0}\left|\left(1+t_{A} g_{0}\right)=<\phi_{A \mathbf{p}}^{-}\right|, \quad g_{A}=g_{0}+g_{0} t_{A} g_{0},
\end{aligned}
$$

where $\phi_{c}$ is localized on the site $A$. The first term describes the direct photoemission process, the second a single elastic scattering, and so on. Let us write the $j$ th term of eq. (3.6) as $Z_{j}(j=1,2,3, \ldots)$. The photoemission intensity is now given at instantaneous atomic configuration $Q$,

$$
\begin{aligned}
& \left|<f_{\mathbf{p}}^{-}\right| \Delta_{\mathbf{k} s}\left|\phi_{c}>\right|^{2}=\left|Z_{1}+Z_{2}+Z_{3}+\ldots\right|^{2}=A+B+C+D+\ldots \\
& A=\left|Z_{1}\right|^{2}, B=2 \operatorname{Re}\left(Z_{1}^{*} Z_{2}\right), C=\left|Z_{2}\right|^{2}, D=2 \operatorname{Re}\left(Z_{1}^{*} Z_{3}\right), \ldots
\end{aligned}
$$

We then take thermal average over all phonon states. The direct term $A$ has no effects from thermal motion, whereas other terms $B, C, \ldots$ are destructively influenced by the thermal motion: Debye-Waller factors suppress the photoelectron diffraction effects $[11,42]$. Of course the core displacement should have some influence on the diffraction terms $B, C, D, \ldots$, however the DW factors have to be more important. In recent high-energy XPS $\left(\omega_{k}>5 \mathrm{keV}\right)$ analyses, we find that recoil effects in the direct term $A$ are affected by phonon excitation [13-16].

\section{Concluding Remarks}

Some important phonon effects observed in core level XAFS and X-ray photoemission spectra are discussed on the basis of nonequilibrium Green's function theory. This theoretical framework allows us to incorporate phonon effects, such as Debye-Waller factors, Franck-Condon factors and electron-phonon interactions in a natural way. The core hole effects are taken into account in the lesser Green's function $g^{<}$, and the excited electron propagation is described by the greater Green's function $g^{>}$.

In the case of deep core excitation, the thermally displaced $1 s$ core function induces $p$ component which plays an important role for pre-edge transitions. In addition to the $1 s \rightarrow 3 d$ E2 transitions, the thermally induced " $1 p$ " $\rightarrow 3 d$ E1 transitions are allowed, which should be sensitive to the temperature. In EXAFS region, these thermal displacement plays a very minor role because $1 s \rightarrow \varepsilon p$ E1 transitions are predominant. This thermally displaced core function is also responsible for the temperature dependent binding energy shift. 
In XPS spectra excited from deep core levels, those thermally induced " $p$ " orbitals have significant influence on the photoemission angular distribution. Even though excited from $1 s$ levels, the angular distribution, where scatterings from surrounding atoms are neglected, does not show $\cos ^{2} \theta$ angular dependence: The deviation is prominent for the heavier atoms because the deep $1 \mathrm{~s}$ orbitals are strongly localized.

\section{Acknowledgment}

The authors are grateful to useful comments on this manuscript by M. Kazama. Parts of this work have been funded by European FP7 MSNano network under Grant No. PIRSES-GA-2012-317554, and by COST Action MP1306 EUSpec. One of the authors $(\mathrm{KN})$ is grateful to the financial support by the CareerSupport Program for Woman Scientist at Chiba University. TF is grateful to the financial support from a Grant-in-Aid for Scientific Research from Ministry of Education, Science and Culture of Japan, Project No. 25246041.

\section{A Displaced Core Functions}

Let first evaluate the Fourier transform of the core function $\phi_{c}$ given in eq. (2.5).

$$
\begin{aligned}
& \int \phi_{L_{c}}(\mathbf{r}) \exp (-i \mathbf{k} \cdot \mathbf{r}) d \mathbf{r}=4 \pi i^{-l_{c}} f_{l_{c}}(k) Y_{L_{c}}(\hat{\mathbf{k}}), \\
& f_{l_{c}}(k)=\int_{0}^{\infty} j_{l_{c}}(k r) R_{l_{c}}(r) r^{2} d r
\end{aligned}
$$

When the atomic position is displaced from the equilibrium position with $\mathbf{u}$, the core function is thus given by

$$
\begin{aligned}
& \phi_{L_{c}}(\mathbf{r}-\mathbf{u})=\int \frac{d \mathbf{k}}{(2 \pi)^{3}} 4 \pi i^{-l_{c}} f_{l_{c}}(k) Y_{L_{c}}(\hat{\mathbf{k}}) \exp [i \mathbf{k} \cdot(\mathbf{r}-\mathbf{u})] \\
& =8 \sum_{L L^{\prime}} i^{l-l^{\prime}-l_{c}} G\left(L_{c} L^{\prime} \mid L\right) Y_{L}(\hat{\mathbf{r}}) Y_{L^{\prime}}^{*}(\hat{\mathbf{u}}) K\left(l_{c}, l, l^{\prime} ; r, u\right), \\
& K\left(l_{c}, l, l^{\prime} ; r, u\right)=\int_{0}^{\infty} d k k^{2} f_{l_{c}}(k) j_{l}(k r) j_{l^{\prime}}(k u),
\end{aligned}
$$

where $G\left(L L^{\prime} \mid L^{\prime \prime}\right)$ is the Gaunt integral defined by $\int Y_{L}(\hat{\mathbf{r}}) Y_{L^{\prime}}(\hat{\mathbf{r}}) Y_{L^{\prime \prime}}^{*}(\hat{\mathbf{r}}) d \hat{\mathbf{r}}$.

For $1 s$ and $2 p$ core functions the radial parts are explicitly given by 


$$
R_{1 s}(r)=2 a^{3 / 2} \exp (-a r), R_{2 p}(r)=\frac{2 a^{5 / 2}}{\sqrt{3}} r \exp (-a r),
$$

which yields $f_{1 s}(k)$ and $f_{2 p}(k)$ as

$$
\begin{aligned}
f_{1 s}(k) & =\frac{4 a^{5 / 2}}{\left(k^{2}+a^{2}\right)^{2}}, \\
f_{2 p}(k) & =\frac{16 a^{7 / 2} k}{\sqrt{3}\left(k^{2}+a^{2}\right)^{3}},
\end{aligned}
$$

By use of these explicit formulas, we can calculate the integral $K\left(l_{c}, l, l^{\prime} ; r, u\right)$. In order to apply residue analyses, we should extend the integral range from $(0, \infty)$ to $(-\infty, \infty)$. We notice that

$$
\begin{aligned}
& j_{l}(k r)=(-1)^{l} j_{l}(-k r), f_{1 s}(k)=f_{1 s}(-k), \\
& f_{2 p}(k)=-f_{2 p}(-k) .
\end{aligned}
$$

The non-zero Gaunt integrals $G\left(L_{c} L \mid L^{\prime}\right)$ in eq. (A3) are restricted to the terms when $l_{c}+l+l^{\prime}=$ even and $\left|l-l_{c}\right| \leq l^{\prime} \leq l+l_{c}$. For the K-edge excitation $\left(l_{c}=0\right), l$ and $l^{\prime}$ should have the same parity. We thus understand that $f_{0}(k) j_{l}(k r) j_{l^{\prime}}(k u)$ is an even function of $k$. In the same way $f_{1}(k) j_{l}(k r) j_{l^{\prime}}(k u)$ is also an even function of $k$. The integral is thus written

$$
K\left(l_{c}, l, l^{\prime} ; r, u\right)=\frac{1}{4} \int_{-\infty}^{\infty} d k f_{l_{c}}(k)\left[h_{l}(k r)+h^{*}(k r)\right] j_{l^{\prime}}(k u) k^{2}
$$

As $|z| \rightarrow \infty, h_{l}(z) \rightarrow i^{-l-1} \exp (i z) / z$, so that $h_{l}(k r) j_{l^{\prime}}(k u)$ therefore decreases exponentially as $|k| \rightarrow \infty$ on the upper half complex plane as far as $r>u$. We can therefore add a large semi-circle to the contour in the upper half-plane, and apply Cauchy's theorem. We should note that $h_{l}(k r) j_{l^{\prime}}(k u) k^{2}$ behaves as $k^{l^{\prime}-l+1}$ at small $k$. At $k=0$ we can have an additional pole. As shown below only $\left|l-l^{\prime}\right| \leq 1$ have finite contribution, so that we have no need to worry about the singularity at $k=0$. For $1 s$ excitation, only $i a$ is the 2 nd order pole in the upper half-plane. The integral is thus given by

$$
\begin{aligned}
& \int_{-\infty}^{\infty} \frac{k^{2}}{\left(k^{2}+a^{2}\right)^{2}} h_{l}(k r) j_{l^{\prime}}(k u) d k \\
& =2 \pi i\left[\frac{d}{d k}\left(\left(\frac{k}{k+i a}\right)^{2} h_{l}(k r)\right) j_{l^{\prime}}(k u)+\left(\frac{k}{k+i a}\right)^{2} h_{l}(k r) \frac{d}{d k} j_{l^{\prime}}(k u)\right]_{k=i a}
\end{aligned}
$$


As $u$ is much smaller than $r$, the ratio of the first term to the second is in the order of $1 /\left(a \sigma_{A}\right)$ which is typically much larger than 1 . We thus keep only the first term of eq. (A9). In the same way, we close the contour in the lower half-plane in the term containing $h_{l}^{*}(k r) j_{l^{\prime}}(k u)$ in eq. (A8). With the help of the identity $h_{l}^{*}(-z)=(-1)^{l} h_{l}(z)$, we finally obtain

$$
\phi_{1 s}(\mathbf{r}-\mathbf{u})=\sum_{L} \phi_{L}^{1 s}(\mathbf{r}) J_{L, 00}(\mathbf{u})
$$

where

$$
\begin{aligned}
& \phi_{L}^{1 s}(\mathbf{r})=i^{l-l_{c}} R_{l}^{1 s}(r) Y_{L}(\hat{\mathbf{r}}), \\
& R_{l}^{1 s}(r)=2 a^{3 / 2}\left[(l+1) h_{l}(z)-z h_{l+1}(z)\right], \quad(z=i a r)
\end{aligned}
$$

The important matrix which describes the atomic displacement is given in terms of spherical harmonics

$$
J_{L L_{c}}(\mathbf{u})=\int Y_{L}^{*}(\hat{\mathbf{r}}) \exp (-a \hat{\mathbf{r}} \cdot \mathbf{u}) Y_{L_{c}}(\hat{\mathbf{r}}) d \hat{\mathbf{r}}
$$

It is important to check that $J_{L L_{c}}(\mathbf{0})=\delta_{L L_{c}}$ and $\phi_{00}^{1 s}(\mathbf{r})=\phi_{1 s}(\mathbf{r})$, which is easy to be proved.

For $2 \mathrm{p}$ excitation, the integral $K\left(1, l, l^{\prime} ; r, u\right)$ is calculated in the same way as eq.(A.9). Here $f_{1}(k)$ has a third order pole at $i a$ in the upper half plane. The integral is thus approximated as

$$
\begin{aligned}
& \int_{-\infty}^{\infty} \frac{k^{3}}{\left(k^{2}+a^{2}\right)^{3}} h_{l}(k r) j_{l^{\prime}}(k u) d k \\
& \approx \pi i\left[\frac{d^{2}}{d k^{2}}\left(\frac{k^{3}}{(k+i a)^{3}} h_{l}(k r)\right) j_{l^{\prime}}(k u)\right]_{k=i a}
\end{aligned}
$$

which yields a relation

$$
\phi_{2 p, m_{c}}(\mathbf{r}-\mathbf{u})=\sum_{L} \phi_{L}^{2 p}(\mathbf{r}) J_{L, 1 m_{c}}(\mathbf{u})
$$

The new functions $\phi_{L}^{2 p}(\mathbf{r})$ are explicitly given by

$$
\begin{aligned}
& \phi_{L}^{2 p}(\mathbf{r})=i^{l-l_{c}} R_{l}^{2 p}(r) Y_{L}(\hat{\mathbf{r}}), \\
& R_{l}^{2 p}(r)=-\frac{2 i}{\sqrt{3}} a^{3 / 2}\left[\left(l^{2}-1-z^{2}\right) h_{l}(z)+z h_{l-1}(z)\right], \\
& (z=i a r) .
\end{aligned}
$$


Explicit formulas of $R_{1}^{1 s}, R_{0}^{2 p}$ and $R_{2}^{2 p}$ are shown in terms of $\rho=a r$

$$
\begin{aligned}
& R_{1}^{1 s}(r)=-2 i a^{3 / 2}\left(1+1 / \rho+1 / \rho^{2}\right) \exp (-\rho), \\
& R_{0}^{2 p}(r)=-\frac{2 i a^{3 / 2}}{\sqrt{3}}(-\rho+1+1 / \rho) \exp (-\rho), \\
& R_{2}^{2 p}(r)=\frac{-2 i a^{3 / 2}}{\sqrt{3}}\left(\rho+2+5 / \rho+9 / \rho^{2}+9 / \rho^{3}\right) \exp (-\rho) .
\end{aligned}
$$

\section{References}

[1] T. Fujikawa, J. Phys. Soc. Jpn. 65 (1996) 87.

[2] T. Fujikawa, J. Electron Spect. Relat. Phenom. 79 (1996) 25.

[3] H. Arai, N. Ueno, T. Fujikawa, X-ray Absorption Fine Structure-XAFS13, CP882, ed. B. Hedman and P. Pianetta, (2007) p. 108.

[4] A. L. Ankudinov, J. J. Rehr, Physica Scripta T115 (2005) 24.

[5] S. Nozawa, T. Iwazumi, H. Osawa, Phys. Rev. B 72 (2005) 121101 (R).

[6] D. Manuel, D. Cabaret, Ch. Brouder, Ph. Sainctavit, A. Bordage, N. Trcera, Phys. Rev. B 85 (2012) 224108.

[7] D. Cabaret, Ch. Brouder, J. Phys. Conference 190 (2009) 012003.

[8] D. Cabaret, Ch. Brouder, A. Juhin, Ph. Sainctavit, Phys. Rev. B 81 (2010) 115125.

[9] L. Plucinski, J. Minar, B. C. Sell, J. Braun, H. Ebert, C. M. Schneider, C. S. Fadley, Phys. Rev. B 78 (2008) 035108.

[10] J. Osterwalder, T. Greber, S. Hüfner,L. Schlapbach, Phys. Rev. Lett. 64 (1990) 2683.

[11] C. S. Fadley, Synchrotron Radiation Research, Advances in Surface and Interface Science, Vol.1 (Plenum, New York, 1992) p.421.

[12] M. A. Vicente Alvarez, H. Ascolani, G. Zampieri, Phys. Rev. B 54 (1996) 14703. 4108.

[13] Y. Takata, Y. Kayanuma, M. Yabashi, M. Tamasaka, Y. Nishino, D. Miwa, Y. Harada, S. Shin, S. Sanaka, E. Ikenaga, K. Kobayashi, Y. Senba, H. Obashi, T. Ishikawa, Phys. Rev. B 75 (2007) 233404.

[14] R. Suzuki, H. Arai, H. Shinotsuka, T. Fujikawa, e-J. Surf. Sci. Nanotech. 3 (2005) 373. 
[15] T. Fujikawa, R. Suzuki, L. Kövér, J. Electron Spect. Relat. Phenom. 151 (2006) 170.

[16] T. Fujikawa, H. Arai, R. Suzuki, H. Shinotsuaka, L. Köver, N. Ueno, J. Electron. Spect. Relat. Phenom. 162 (2008) 146.

[17] M-H. Shang, T. Fujikawa, N. Ueno, e-J. Surf. Sci. Nanotechnol. 10 (2012) 128.

[18] M-H. Shang, T. Fujikawa, N. Ueno, Anal. Chem. 85 (2013) 3739.

[19] U. Gelius, K. Siegbahn, J. Chem Soc. Faraday Discuss. 54 (1972) 257.

[20] C-O. Almbladh, L. Hedin, in E.E. Koch ( Eds.) Handbook on Synchrotron Radiation ( North-Holland), Vol.1b, p.607 .

[21] R. Matzdorf, Surf. Sci. Rep. 30 (1998) 153.

[22] T. Cuk, D. H. Lu, X. J. Zhou, Z-X. Shen, T. P. Devereaux, N. Nagaosa, Phys. Stat. Sol. (b) 242 (2005) 11.

[23] T, Fujikawa, H. Arai, J. Electron Spect. Relat. Phenom. 174 (2009) 85.

[24] T. Fujikawa, H. Arai, J. Elect. Spect. Relat. Phenom. 123 (2002) 19.

[25] G. Baym, Ann. Phys. 14 (1962) 1.

[26] L. Hedin, S. Lundqvist, Solid State Physics (Acadimic Press, New York, 1969) Vol. 23, p.1 eds. F. Seitz, D. Turnbull and H. Ehrenreich

[27] J. Braun, J. Minar, S. Mankovsky, S. N. Strocov, N. B. Brookes, L. Plucinski, C. M. Schneider, C. S. Fadley, H. Ebert, Phys. Rev. B 88 (2013) 205409.

[28] T. Fujikawa, J. Phys. Soc. Jpn. 68 (1999) 2444.

[29] T. Fujikawa, J. Synchrotron Rad. 8 (2001) 76.

[30] N. D. Mermin, J. Math. Phys. 7 (1966) 1038.

[31] T. Fujikawa, J. J. Rehr, Y. Wada and S. Nagamatsu, J. Phys. Soc. Jpn. 68 (1999) 1259.

[32] T. Fujikawa, J.J. Rehr, Y. Wada, S. Nagamatsu, J. Synchrotron Rad. 6 (1999) 317.

[33] C. Brouder and J. Goulon, Physica B 158 (1989) 351.

[34] J. Schwinger, Phys. Rev. 51 (1946) 651.

[35] R. M. Wilcox, J. Math. Phys. 8 ( 1967) 962.

[36] C. Caroli, D. Ledeer-Rozenblatt, B. Roulet, D. Saint-James, Phys. Rev. B 8 (1973) 4552.

[37] M. Kazama, H. Shinotsuka, Y. Ohori, K. Niki, T. Fujikawa, L. Kövér, Phys. Rev. B 89 (2014) 045110. 
[38] K. Niki, N. Yamamura, Y. Ohori, M. Kazama, T. Fujikawa, L. Kövér, Surf. Interface Anal. 46 (2014) 924.

[39] T. Fujikawa, J. Phys. Soc. Jpn. 50 (1981) 1321.

[40] T. Fujikawa, J. Phys. Soc. Jpn. 51 (1982) 251.

[41] T. Fujikawa, J. Phys. Soc. Jpn. 54 (1985) 2747.

[42] T. Yanagawa, T. Fujikawa, J. Phys. Soc. Jpn. 65 (1996) 1832. 


\section{Figure Caption}

Fig.1 The temperature dependence of the pre-edge intensities for Ti K-edge excitation for different thermal factors $\sigma_{0}^{2}$ : (a) $1 \times 10^{-2}$ a.u. ${ }^{2}$, (b) $1 \times 10^{-3}$ a.u. ${ }^{2}$, (c) $1 \times 10^{-4} a . u .^{2}$ and (d) $1 \times 10^{-5} a . u .^{-2}$. Both E1 $(1 s \rightarrow n p)$ and E2 $(1 s \rightarrow n d)$ transitions are shown.

Fig.2 Temperature dependence of the binding energy shifts at Ti K-edge (see eq. (2.60)) for different energy ratio $\beta=\sum_{m} \varepsilon_{m} / \varepsilon_{0}$.

Fig.3 The photoelectron angular distribution excited from Si K-edge at $\varepsilon_{p}=100 \mathrm{eV}$ : In (a) $\sigma_{0}^{2}=0.005 a . u .^{2}$ and $0.01 a . u .^{2}$ in (b) for various temperatures $\mathrm{T}$ from $10 \mathrm{~K}$ to $300 \mathrm{~K}$. For simplicity we neglect elastic scatterings from surrounding atoms. We normalize the intensity so that the integrated intensity over $\theta$ to be the same.

Fig. 4 The photoelectron angular distribution excited from Ti K-edge at $\varepsilon_{p}=100 \mathrm{eV}$ : In (a) $\sigma_{0}^{2}=0.005 a . u .^{2}$ and $0.01 a . u .^{2}$ in (b) for various temperatures $\mathrm{T}$ from $10 \mathrm{~K}$ to $300 \mathrm{~K}$. For simplicity we neglect elastic scatterings from surrounding atoms. We normalize the intensity so that the integrated intensity over $\theta$ to be the same.

Fig.5 The photoelectron angular distribution excited from $\mathrm{Cu}$ K-edge at $\varepsilon_{p}=100 \mathrm{eV}$ : In (a) $\sigma_{0}^{2}=0.001 a . u .^{2}$ and $0.005 a . u .^{2}$ in (b) for various temperatures $\mathrm{T}$ from $10 \mathrm{~K}$ to $300 \mathrm{~K}$. For simplicity we neglect elastic scatterings from surrounding atoms. We normalize the intensity so that the integrated intensity over $\theta$ to be the same. 


\section{Figure}
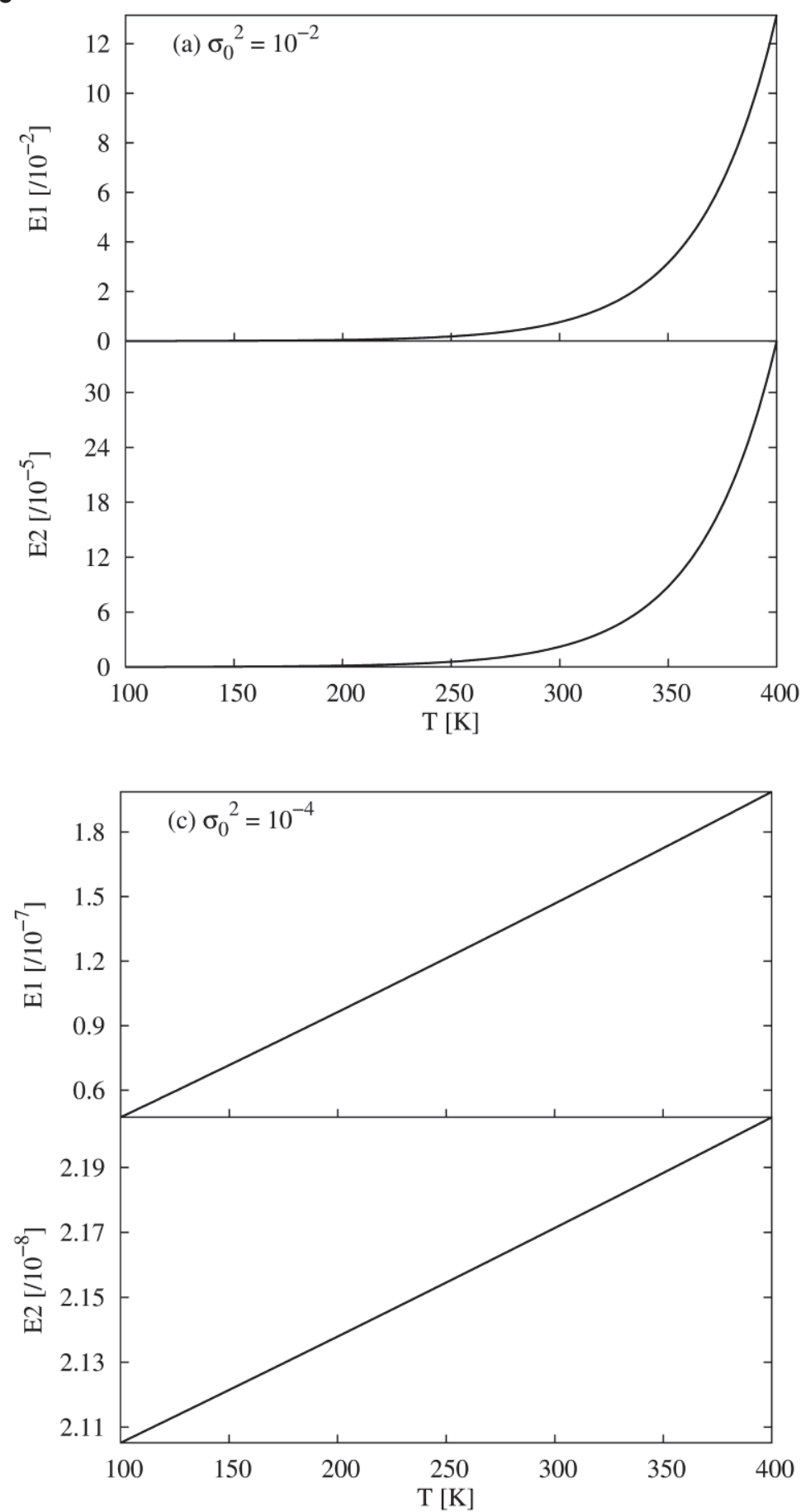
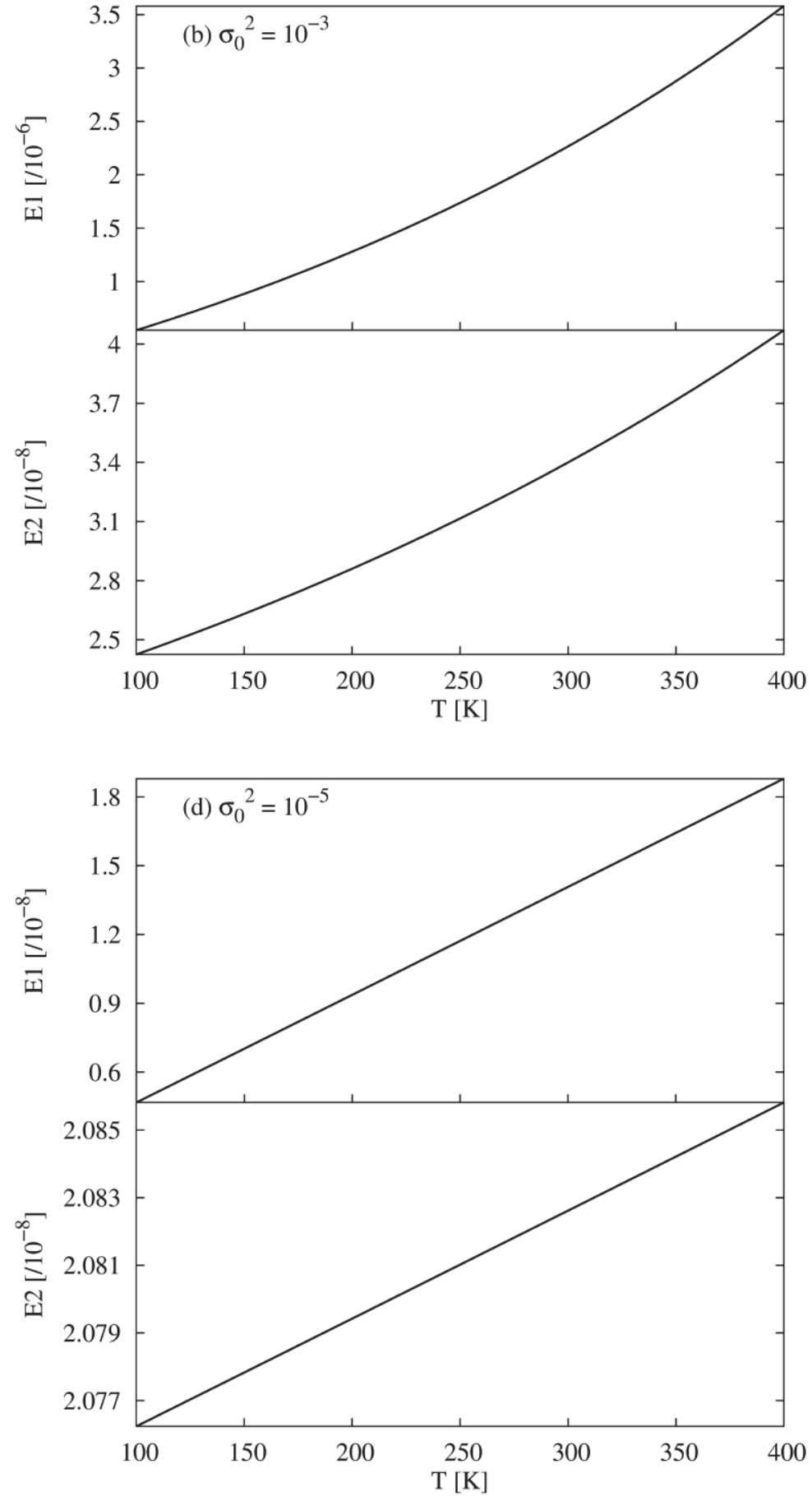


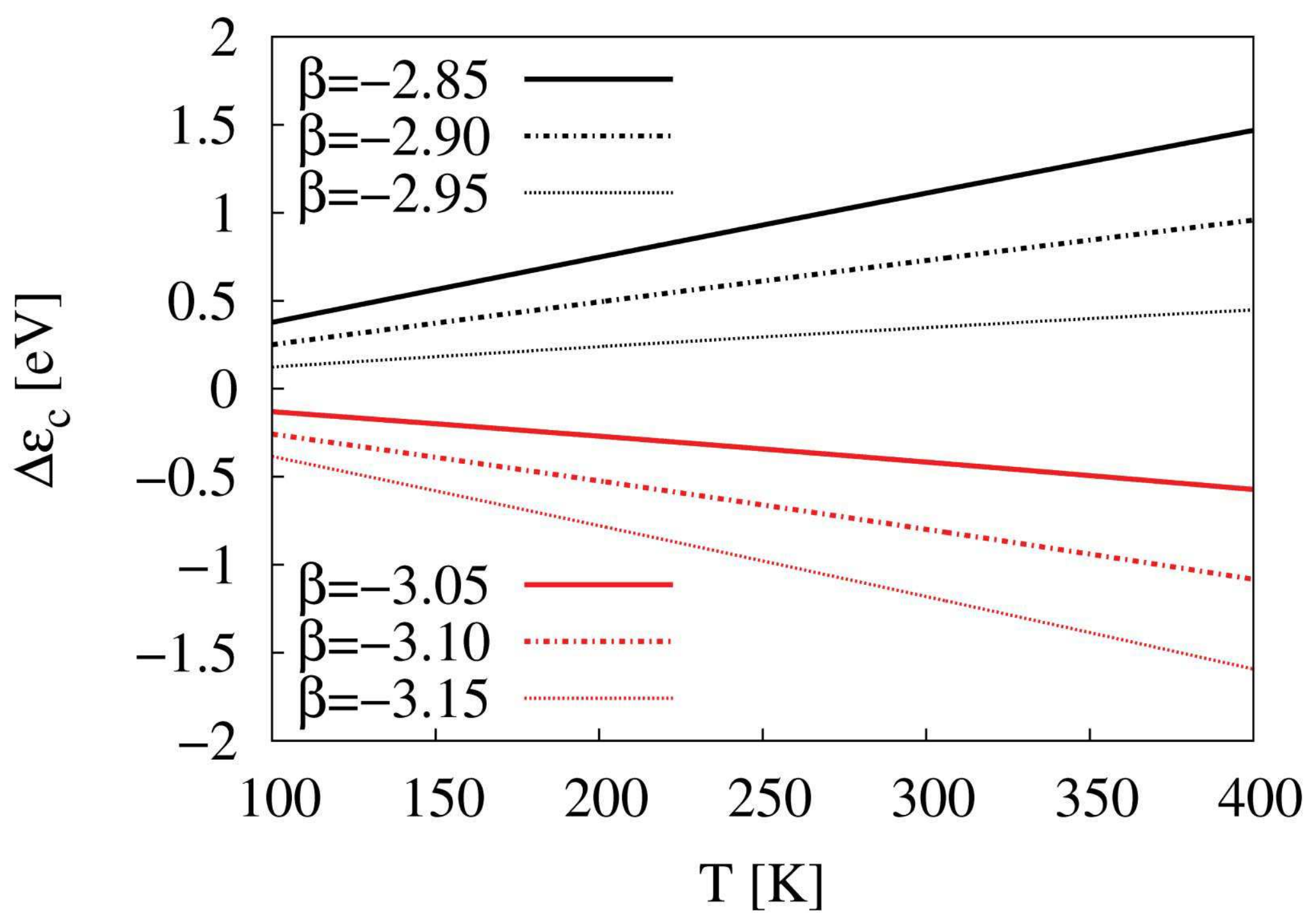




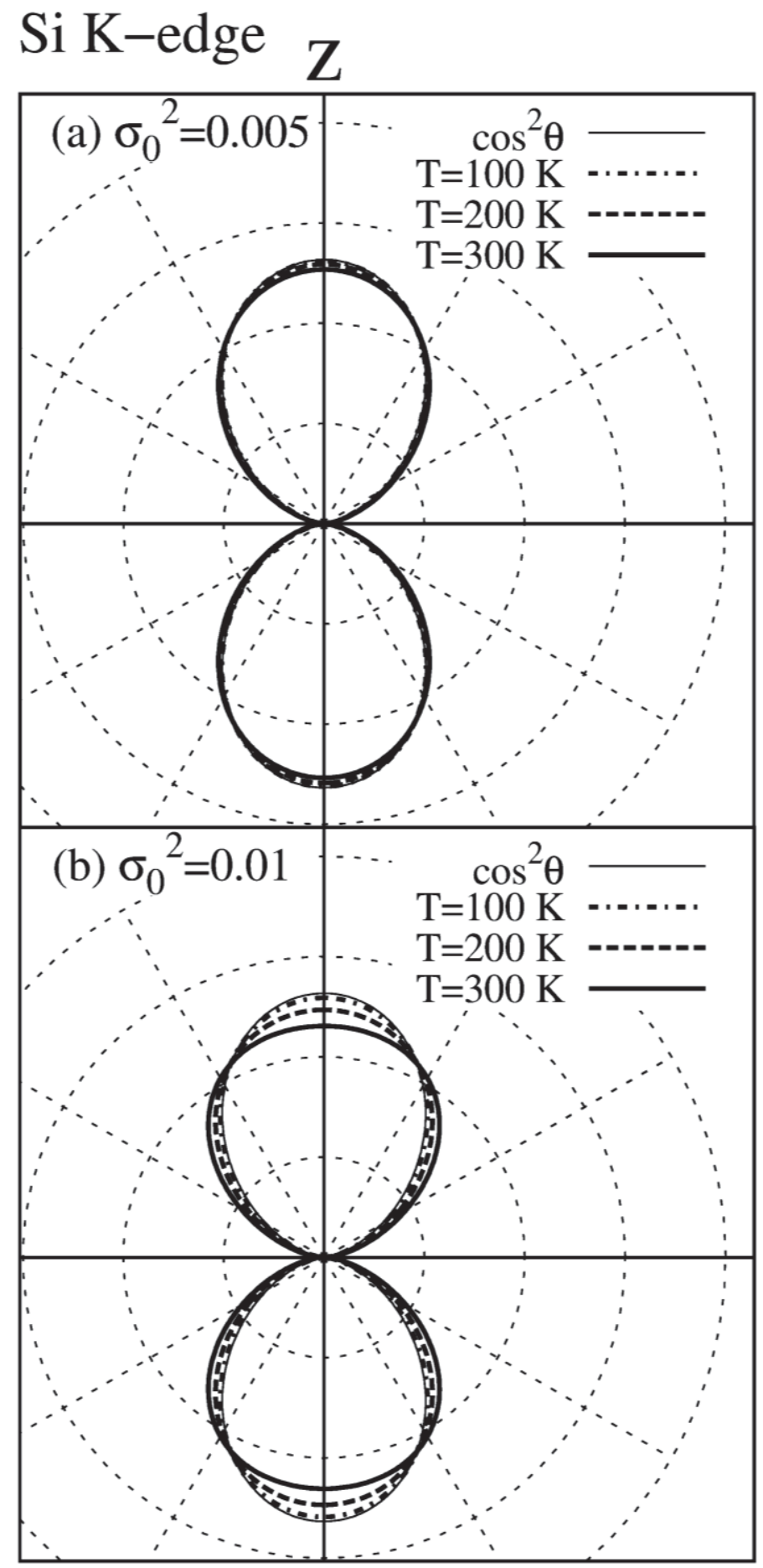




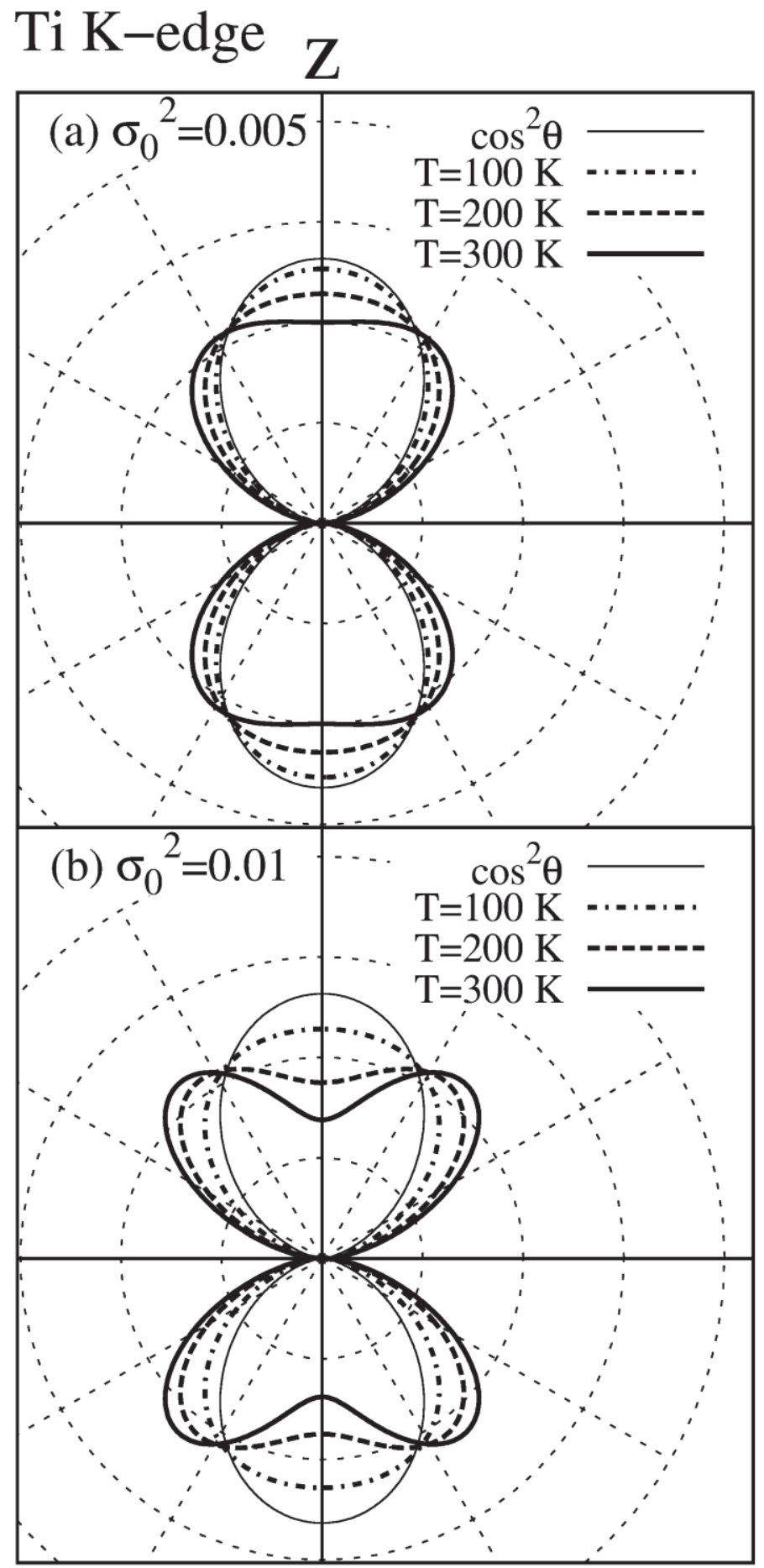




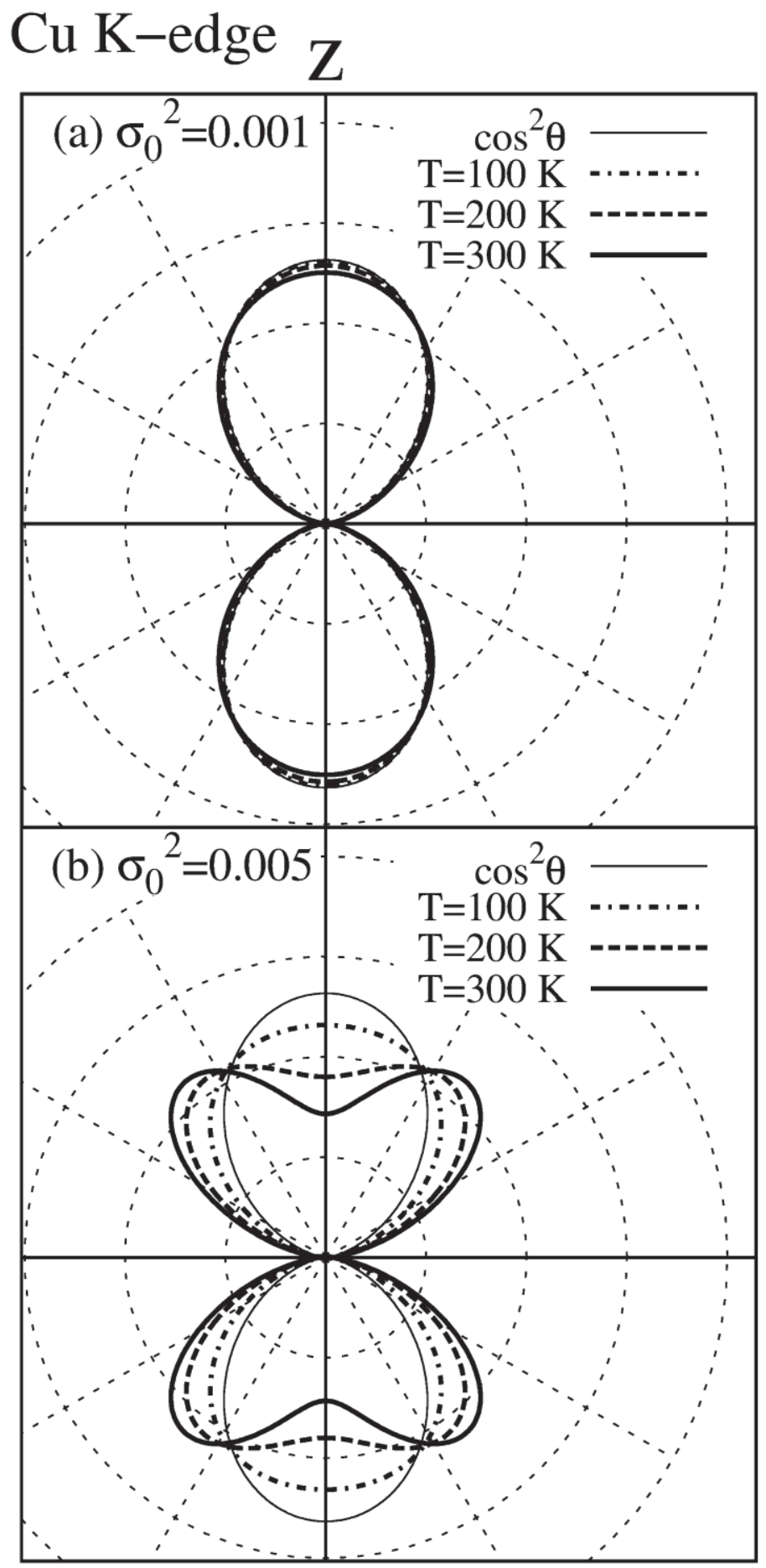

